\title{
Prevalence of BRCA1/BRCA2 mutations in a Brazilian population sample at-risk for hereditary breast cancer and characterization of its genetic ancestry
}

Gabriela C. Fernandes ${ }^{1, *}$, Rodrigo A.D. Michelli2,*, Henrique C.R. Galvão2,* André E. Paula ${ }^{1}$, Rui Pereira ${ }^{3,4}$, Carlos E. Andrade ${ }^{2}$, Paula S. Felicio ${ }^{5}$, Cristiano P. Souza ${ }^{2}$, Deise R.P. Mendes ${ }^{1}$, Sahlua Volc ${ }^{2}$, Gustavo N. Berardinelli ${ }^{1}$, Rebeca S. Grasel ${ }^{2}$, Cristina S. Sabato ${ }^{1}$, Danilo V. Viana ${ }^{2}$, José Carlos Machado ${ }^{4}$, José Luis Costa ${ }^{4}$, Edmundo C. Mauad ${ }^{2,6}$, Cristovam Scapulatempo-Neto ${ }^{1,5,7}$, Banu Arun ${ }^{8}$, Rui M. Reis $^{1,2,5,9,10}$, Edenir I. Palmero ${ }^{1,2,5,11}$

${ }^{1}$ Center of Molecular Diagnosis, Barretos Cancer Hospital, Barretos, São Paulo, Brazil

${ }^{2}$ Oncogenetics Department, Barretos Cancer Hospital, Barretos, São Paulo, Brazil

${ }^{3}$ Institute of Research and Innovation in Health, University of Porto, Porto, Portugal

${ }^{4}$ Institute of Molecular Pathology and Immunology at the University of Porto (IPATIMUP), Porto, Portugal

${ }^{5}$ Molecular Oncology Research Center, Barretos Cancer Hospital, Barretos, São Paulo, Brazil

${ }^{6}$ Prevention Department, Barretos Cancer Hospital, Barretos, São Paulo, Brazil

${ }^{7}$ Pathology Department, Barretos Cancer Hospital, Barretos, São Paulo, Brazil

${ }^{8}$ MD Anderson Cancer Center, Houston, Texas, USA

${ }^{9}$ Life and Health Sciences Research Institute (ICVS), Health Sciences School, University of Minho, Braga, Portugal

${ }^{10}$ ICVS/3B's-PT Government Associate Laboratory, Braga/Guimarães, Portugal

${ }^{11}$ Barretos School of Health Sciences, Dr. Paulo Prata-FACISB, São Paulo, Brazil

*These authors contributed equally to this work

Correspondence to: Edenir I. Palmero, email: edenirip@yahoo.com.br

Keywords: hereditary breast cancer, BRCA1/BRCA2 mutation profile in Brazil, genetic ancestry, HBOC in brazil, c.5266dupC prevalence in brazil

Received: April 04, $2016 \quad$ Accepted: October 01, $2016 \quad$ Published: October 12, 2016

\section{ABSTRACT}

Background: There are very few data about the mutational profile of families at-risk for hereditary breast and ovarian cancer (HBOC) from Latin America (LA) and especially from Brazil, the largest and most populated country in LA.

Results: Of the 349 probands analyzed, $21.5 \%$ were BRCA1/BRCA2 mutated, $65.3 \%$ at $B R C A 1$ and $34.7 \%$ at BRCA2 gene. The mutation c.5266dupC (former 5382insC) was the most frequent alteration, representing $36.7 \%$ of the $B R C A 1$ mutations and $24.0 \%$ of all mutations identified. Together with the BRCA1 c.3331_3334delCAAG mutation, these mutations constitutes about $35 \%$ of the identified mutations and more than $50 \%$ of the BRCA1 pathogenic mutations. Interestingly, six new mutations were identified. Additionally, 39 out of the 44 pathogenic mutations identified were not previously reported in the Brazilian population. Besides, 36 different variants of unknown significance (VUS) were identified. Regarding ancestry, average ancestry proportions were $70.6 \%$ European, $14.5 \%$ African, $8.0 \%$ Native American and 6.8\% East Asian.

Materials and methods: This study characterized 349 Brazilian families at-risk for HBOC regarding their germline BRCA1/BRCA2 status and genetic ancestry.

Conclusions: This is the largest report of BRCA1/BRCA2 assessment in an atrisk HBOC Brazilian population. We identified $21.5 \%$ of patients harboring BRCA1/ $B R C A 2$ mutations and characterized the genetic ancestry of a sample group at-risk for 


\section{hereditary breast cancer showing once again how admixed is the Brazilian population. No association was found between genetic ancestry and mutational status. The knowledge of the mutational profile in a population can contribute to the definition of more cost-effective strategies for the identification of HBOC families.}

\section{INTRODUCTION}

Breast cancer $(\mathrm{BC})$ constitutes the leading cause of cancer mortality among Brazilian women. According to the Brazilian National Cancer Institute (INCA), the number of new BC cases expected in 2014 was 57,120. A higher incidence occurs in Southeastern Brazil, with an estimated risk of 71 new cases/year per 100,000 women [1].

It is estimated that 5 to $10 \%$ of the $\mathrm{BC}$ cases are hereditary $[2,3]$. Therefore, in Brazil, approximately 6,000 new cases of hereditary BC are expected yearly, which is alarming both for its numerical proportions, and for the fact that most of these tumors are not recognized as from an hereditary origin $[1,2]$.

In Brazil, there are few services specialized in identifying and monitoring families at-risk for hereditary cancer. These services are mainly concentrated in capital cities of some Brazilian states, limiting or at least hindering the access of a great share of the population living in more remote areas [4-6]. Thus, data on $\mathrm{BC}$ familial aggregates are still scarce in Brazil. The main studies published so far involve i) specific populations, such as young women with $\mathrm{BC}$ [7]; or ii) regions and/or specific gene mutations, with most studies focusing their analysis on the founder mutations at BRCA1/BRCA2 genes [7-13].

\section{Genetic ancestry}

The ethnic substructure of the population is an important factor that can influence the incidence, prognosis and mortality of BC. A large body of evidence suggested an inverse correlation between low incidence of $\mathrm{BC}$ and high rates of mortality among African-American women when compared with Caucasian, with the risk of death from BC being 67\% higher among African-Americans [14-16]. In addition, work developed in Mexico by Fejerman and collaborators [17], showed that for every $25 \%$ increase in European ancestry there was a $20 \%$ increase in risk of BC. Otherwise, results of a case-control study published by Bonilla and collaborators [18] involving 328 Uruguayan women showed no evidence that overall genetic ancestry differs between $\mathrm{BC}$ patients and controls in Uruguay.

According to Kehdy and collaborators, Brazil is a classical model for population genetics studies on admixture, since it received several immigration waves from diverse European origins during the last five centuries. In addition, African slaves arrived to Brazil during four centuries, and the geographic origin of Brazilian slaves differ from Caribbean and African American [19, 20]. However, little is known about the genetic ancestry profile in a highly admixed population with $\mathrm{BC}$ and/or ovarian cancer (OC). In addition, to the best of our knowledge, there are no studies characterizing the ancestry profile of a cohort at-risk for hereditary BC in the Brazilian population.

Therefore, we characterized a cohort of 349 families at-risk for hereditary breast and ovary cancer (HBOC) from a single institution for the presence of germline $B R C A 1$ and $B R C A 2$ mutations, as well as for ethnic composition (genetic ancestry) and correlated these molecular findings with the clinical and familial history of patients.

\section{RESULTS}

\section{General characterization}

Among the 349 index cases analyzed (following criteria described in the "Material and Methods" section), $97.4 \%(n=340)$ were female and $2.6 \%(n=9)$ were male, all with breast and/or OC history. The vast majority of probands had BC as the primary tumor $(n=292,83.7 \%)$, $50(14.3 \%)$ had a history of OC and 7 patients $(2.0 \%)$ had other tumors than $\mathrm{BC}$ or OC as primary tumor site (gall bladder $(n=2)$, colorectal $(n=2)$, melanoma $(n=1)$, prostate $(n=1)$ and endometrium $(n=1))$.

The mean age at diagnosis was 41 years $(\mathrm{SD}=12$, CI: $16-86$ years). When categorized, $22.9 \%$ (80 patients) received a cancer diagnosis at an age below 30 years, $32.1 \%(n=112)$ between 30 and 40 years, $24.9 \%$ $(n=87)$ were diagnosed at ages between 40 and 50 years old and $20.1 \%$ (70 patients) of the probands had their primary tumors detected after 50 years old (Supplementary Table S1).

The majority of $\mathrm{BC}$ types were invasive ductal carcinoma $(85.2 \%)$, followed by intraductal carcinoma (7.6\%), papillary $(3.4 \%)$, lobular $(3.4 \%)$ and medular $(1.1 \%)$ carcinomas. As for OC types, most (74\%) were serous adenocarcinomas, followed by undifferentiated tumors $(13 \%)$, endometrioid tumors $(7 \%)$, clear cell tumors $(2 \%)$, mucinous tumors $(2 \%)$ and peritoneal tumors $(2 \%)$.

Among the 349 probands, $21.5 \%$ were germline mutated $(n=75)$ with $65.3 \%$ of them carrying mutations in $B R C A 1$ and $34.7 \%$ in the BRCA2. When segregating mutated patients according to primary tumor site, $20.5 \%$ of patients with $\mathrm{BC}$ were mutated (60 cases) being $63.3 \%$ of the mutations in the BRCA1 gene and $36.7 \%$ in BRCA2. Among patients whose primary tumor was OC, $30.0 \%$ (15 patients) presented pathogenic mutations in the $B R C A 1 / B R C A 2$ genes, with the majority (73.3\%) being located in $B R C A 1$. 
Taking into consideration only mutated patients with $\mathrm{BC}$, among those with $B R C A 1$ mutated, $72.7 \%$ of the tumors were triple negative and $27.3 \%$ Luminal A or B1. Regarding the $B R C A 2$ mutated patients, $9.1 \%$ were triple negative, $63.6 \%$ Luminal A or B1 and 27.3\% Luminal B2. For those $B R C A 1 / B R C A 2 \mathrm{WT}$, the majority (52.6\%) were Luminal A/B1, followed by Luminal B2 (20.8\%), triple negative (18.2\%) and HER2 (8.4\%). Lastly, patients with a VUS on $B R C A 1$ or $B R C A 2$ had a majority of tumors of Luminal A/B1 types (54.7\%), followed by triple negative (19.3\%), Luminal B2 and HER2 (13\% each).

When the age at diagnosis was compared with the mutational status of $B R C A 1 / B R C A 2$, it was observed that, among the group with germline mutations in $B R C A 1$, the mean age at diagnosis was 42.1 years $(\mathrm{SD}=11.1$, $\mathrm{CI}=23-77$ years) and for the group with $B R C A 2$ mutations, the mean age was 44.7 years $(\mathrm{SD}=11.5$, $\mathrm{CI}=26-67$ years) (Supplementary Table S1 and Supplementary Figure S1).

\section{Cancer family history}

Among the 349 families evaluated, 85 (24.5\%) reported the presence of at least one case of OC among first, second or third degree relatives (or in the proband itself), $26(7.5 \%)$ had bilateral breast cancer (BBC), 11 families $(3.2 \%)$ reported cases of male $\mathrm{BC}$, and 18 families $(5.2 \%)$ had history of pancreatic cancer.

We observed an average of three generations affected by cancer $(\mathrm{SD}=5)$, and 5 cancer cases per family $(\mathrm{SD}=6)$, with a mean of $3 \mathrm{BC}$ cases $(\mathrm{SD}=5)$. When considering mutated families, the average of $\mathrm{BC}$ among cases with $B R C A 1$ germline mutations was 2.8 $(\mathrm{SD}=1.8)$, while for $B R C A 2$ mutated cases this mean was 3.4 $(\mathrm{SD}=3.0)$.

There was a statistically significant difference in the distribution of $\mathrm{BBC}$ cases according to the status of $B R C A 1 / B R C A 2$ mutations. When the analysis was stratified by gene, it was observed that the presence of BBC was associated with the presence of germline mutations in BRCA1 ( $p=0.002)$, with $8 / 49$ families with $B R C A 1$ mutation presenting $\mathrm{BBC}$ cases in the family.

\section{Mutational profile}

Among the 349 index cases included in the study, 75 $(21.5 \%)$ showed pathogenic germline mutations in $B R C A 1$ $(n=49)$ or BRCA2 $(n=26)$ (Table 1$)$. No patient carrying more than one pathogenic variant was identified. The distribution of the different types of mutations (missense, nonsense, frameshift, splicing and rearrangements) along the exons as well as its frequency are shown in Figure 1 and Supplementary Figure S2 respectively.

Among the mutated patients, 60 had a personal history of $\mathrm{BC}$ and 15 of $\mathrm{OC}$. About the $\mathrm{BC}$ with a
$B R C A 1$ mutation, the great majority were invasive ductal carcinomas. For BRCA2 mutated patients, $82 \%$ were invasive ductal carcinomas, 14\% lobular invasive carcinomas and $4 \%$ were medular tumors. Regarding OC patients with a BRCA1 mutation, $67 \%$ were high grade serous adenocarcinoma, and $33 \%$ were undifferentiated. For the BRCA2 mutated, the predominant pathology were also the serous adenocarcinoma (50\%), followed by one case of peritoneal tumor and one of undifferentiated ovarian tumor.

For $B R C A 1,21$ different pathogenic mutations were identified and one of those was present in 18 different families, representing $36.7 \%$ of the $B R C A 1$ mutated families: the founder mutation c.5266dupC, previously known as 5382insC, in exon 20 of BRCAl gene. The second most common mutation (shared by 8 families) was the frameshift alteration c.3331_3334delCAAG in exon 11 of $B R C A 1$. These mutations were identified in families coming from different Brazilian regions: 64\% from Southeast, $28 \%$ from Central-West and $8 \%$ came from the North region of the Brazilian territory.

Twenty-two distinct mutations were identified in $B R C A 2$. The only change shared by more than two families was the frameshift mutation c.2808_2811delACAA in exon 11 of this gene, present in three families. Among the identified mutations, two were rearrangements: a deletion of exons 5 to 7 at $B R C A 1$ identified by MLPA analysis and an Alu insertion at BRCA2 exon 3, identified by a mutation-specific PCR.

An association between the presence of c.5266dupC mutation and the occurrence of BBC was observed: $22.7 \%$ of families with this mutation had $\mathrm{BBC}$, in the proband $(16.7 \%)$ or in the family (11.1\%) (OR: 3.987, 95\% CI, 1.210-13.137, $p=0.016)$. Additionally, there was an association between the presence of this mutation and the development of OC (OR: 3.2, 95\% CI, 1.1-9.0) $(p=0.023)$.

Six new mutations were identified (not described in the HGMD, BIC, UMD and ClinVar databases): 2 in BRCA1 (c.1962dupG and c.5161delC) and four in BRCA2 (c.5158_5159insA, c.5216dupA, c.7987delG and c.8711delT). All new mutations identified are frameshift alterations and lead to the formation of an altered and probably non-functional protein. Besides, 39 out of the 44 pathogenic mutations identified in our study $(88.6 \%)$ were not previously reported in the Brazilian population.

Regarding missense variants, there was an extensive review of databases and in silico analysis, in order to classify those alterations according to its possible pathogenicity. As shown in Table 2, there is a large inconsistency among the databases considered for a large proportion of the variants analyzed. In addition, data regarding the frequency with which the change has been identified (number of families with the given alteration) and the co-segregation with proven pathogenic variants is reported. As detailed in Table 3, five variants were 
Table 1: Germline mutations identified (pathogenic)

\begin{tabular}{|c|c|c|c|c|c|c|}
\hline Mutation & $\begin{array}{l}\text { Mutated } \\
\text { Gene }\end{array}$ & $\begin{array}{c}\text { Number } \\
\text { of } \\
\text { families }\end{array}$ & $\begin{array}{l}\text { Mutation } \\
\text { Type }\end{array}$ & $\begin{array}{l}\text { Tumors in index } \\
\text { patient, age at onset* }\end{array}$ & $\begin{array}{l}\text { Number } \\
\text { of } B C \\
\text { cases in } \\
\text { relatives* }\end{array}$ & $\begin{array}{l}\text { Number } \\
\text { of OC } \\
\text { cases in } \\
\text { relatives* }\end{array}$ \\
\hline c.65T $>$ C p.Leu22Ser & $B R C A 1$ & 1 & Missense & Ovarian, 55 & 0 & 0 \\
\hline c. $181 \mathrm{~T}>$ G p.Cys61Gly & $B R C A 1$ & 1 & Missense & Breast, 59 & 5 & 1 \\
\hline c. $188 \mathrm{~T}>$ A p.Leu63Ter & $B R C A 1$ & 1 & Nonsense & Breast, 26 & 4 & 0 \\
\hline $\begin{array}{l}\text { c.470_471delCT } \\
\text { p.Ser157Ter }\end{array}$ & $B R C A 1$ & 1 & Missense & Breast, 43 & 5 & 0 \\
\hline $\begin{array}{l}\text { c. } 1088 \text { delA } \\
\text { p.Asn363Ilefs*11 }\end{array}$ & $B R C A 1$ & 1 & Frameshift & Breast, 36 & 2 & 0 \\
\hline c. $1687 \mathrm{C}>\mathrm{T}$ p.Gln563Ter & $B R C A 1$ & 2 & Nonsense & Breast, 29; Breast, 48 & $4 ; 3$ & $0 ; 0$ \\
\hline $\begin{array}{l}\text { c.1912delG } \\
\text { "p.Glu638Asnfs*13" }\end{array}$ & $B R C A 1$ & 1 & Frameshift & Breast, 53 & 3 & 0 \\
\hline $\begin{array}{l}\text { c.1962dupG } \\
\text { p.Tyr655Valfs*18 }\end{array}$ & $B R C A 1$ & 1 & Frameshift & Breast, 34 & 3 & 0 \\
\hline $\begin{array}{l}\text { c. } 2405 \_2406 \text { delTG } \\
\text { p.Val802Glufs*7 }\end{array}$ & $B R C A 1$ & 1 & Frameshift & Breast, 35 & 2 & 0 \\
\hline c.3239T $>$ A p.Leu1080Ter & $B R C A 1$ & 1 & Nonsense & Breast, 31 & 1 & 0 \\
\hline $\begin{array}{l}\text { c.3331_3334delCAAG } \\
\text { p.Q1111Nfs*5 }\end{array}$ & $B R C A 1$ & 8 & Frameshift & $\begin{array}{c}\text { Breast, 39; Ovarian, 32; } \\
\text { Breast, 32; Breast, 46; } \\
\text { Breast, 47; Breast, 30; } \\
\text { Ovarian, 54; Ovarian, } 53\end{array}$ & $\begin{array}{l}2 ; 1 ; 4 ; 4 \\
3 ; 5 ; 0 ; 0\end{array}$ & $\begin{array}{c}0 ; 0 ; 1 ; 0 ; 2 ; \\
0 ; 0 ; 0\end{array}$ \\
\hline $\begin{array}{l}\text { c.3764dupA } \\
\text { p.Asn1255Lysfs*11 }\end{array}$ & $B R C A 1$ & 1 & Frameshift & Breast, 35 & 3 & 0 \\
\hline $\begin{array}{l}\text { c.3916_3917delTT } \\
\text { p.Leu1306Aspfs*23 }\end{array}$ & $B R C A 1$ & 2 & Frameshift & Ovarian, 52; breast, 32 & $3 ; 1$ & $2 ; 0$ \\
\hline $\begin{array}{l}\text { c. } 4165 \text { 4166delAG } \\
\text { p.Ser1389Ter }\end{array}$ & $B R C A 1$ & 1 & Frameshift & Breast, 33 & 2 & 0 \\
\hline c. $4357+1 \mathrm{G}>\mathrm{C}$ & $B R C A 1$ & 1 & Splicing & Breast, 47 & 3 & 0 \\
\hline $\begin{array}{l}\text { c. } 4964 \text { 4982del19 } \\
\text { p.Ser1655Tyrfs*16 }\end{array}$ & $B R C A 1$ & 1 & Frameshift & Breast, 29 & 2 & 0 \\
\hline $\begin{array}{l}\text { c. } 5030 \text { 5033delCTAA } \\
\text { p.Thr1677Ilefs*2 }\end{array}$ & $B R C A 1$ & 1 & Frameshift & Breast, 40 & 4 & 0 \\
\hline $\begin{array}{l}\text { c.5161delC } \\
\text { p.GIn1721Serfs } * 9\end{array}$ & $B R C A 1$ & 1 & Frameshift & Breast, 45 & 1 & 1 \\
\hline c.5251C $>$ T p.Arg1751Ter & $B R C A 1$ & 1 & Nonsense & Breast, 23 & 4 & 1 \\
\hline $\begin{array}{l}\text { c.5266dupC } \\
\text { p.Gln1756Profs } * 74\end{array}$ & $B R C A 1$ & 18 & Frameshift & $\begin{array}{l}\text { Breast, 36; Breast, 63; } \\
\text { Ovarian, 77; Breast, 36; } \\
\text { Breast, 44; Ovarian, 54; } \\
\text { Ovarian, 47; Ovarian, } \\
\text { 53: Breast, 31; Ovarian, } \\
\text { 47; Breast, 41; Ovarian, } \\
\text { 49; Breast, 33; Breast, } \\
\text { 42; Breast, 37; Breast, } \\
\text { 28; Breast, 28; Breast, } 47\end{array}$ & $\begin{array}{c}8 ; 4 ; 2 ; 2 \\
4 ; 2 ; 0 ; 4 ; \\
2 ; 0 ; 5 ; 0 ; \\
6 ; 3 ; 2 ; 4 ; \\
\quad 1 ; 4\end{array}$ & $\begin{array}{l}0 ; 0 ; 0 ; 1 ; 0 ; \\
0 ; 0 ; 1 ; 0 ; 0 ; \\
0 ; 0 ; 1 ; 0 ; 0 ; \\
\quad 0 ; 0 ; 0\end{array}$ \\
\hline c. $5444 \mathrm{G}>$ A p.Trp1815Ter & $B R C A 1$ & 1 & Nonsense & Breast, 45 & 6 & 0 \\
\hline $\begin{array}{l}\text { c.5463_5464insT } \\
\text { p.His1822Serfs*7 }\end{array}$ & $B R C A 1$ & 1 & Frameshift & Breast, 50 & 4 & 1 \\
\hline
\end{tabular}




\begin{tabular}{|c|c|c|c|c|c|c|}
\hline deletion exons 5 to 7 & BRCA1 & 1 & Rearrangement & Breast, 56 & 2 & 1 \\
\hline c. $2 \mathrm{~T}>\mathrm{G}$ p.Met1Arg & $B R C A 2$ & 2 & Missense & Breast, 48; Breast, 43 & $6 ; 5$ & $0 ; 0$ \\
\hline c.156_157insAlu & $B R C A 2$ & 1 & Rearrangement & Breast, 29 & 2 & 0 \\
\hline $\begin{array}{l}\text { c.658_659delGT } \\
\text { p.Val220Ilefs*4 }\end{array}$ & $B R C A 2$ & 1 & Frameshift & Breast, 49 & 2 & 1 \\
\hline $\begin{array}{l}\text { c.1138delA } \\
\text { p.Ser380Valfs*19 }\end{array}$ & $B R C A 2$ & 1 & Frameshift & Breast, 49 & 4 & 0 \\
\hline $\begin{array}{l}\text { c. } 2808 \text { 2811delACAA } \\
\text { p.Ala938Profs*21 }\end{array}$ & $B R C A 2$ & 3 & Frameshift & $\begin{array}{c}\text { Breast, } 43 \text {; Breast, } 34 ; \\
\text { Breast, } 36\end{array}$ & $2 ; 4 ; 2$ & $0 ; 0 ; 0$ \\
\hline $\begin{array}{l}\text { c. } 3858 \text { 3860delAAA } \\
\text { p.Lys1286del }\end{array}$ & $B R C A 2$ & 1 & $\begin{array}{l}\text { In frame } \\
\text { deletion }\end{array}$ & Breast, 49 & 2 & 0 \\
\hline $\begin{array}{l}\text { c.4284dupT } \\
\text { p.Gln1429Serfs*9 }\end{array}$ & $B R C A 2$ & 1 & Frameshift & Ovarian, 65 & 0 & 0 \\
\hline $\begin{array}{l}\text { c. 5073dupA } \\
\text { p.Trp1692Metfs*3 }\end{array}$ & $B R C A 2$ & 2 & Frameshift & Ovarian, 63; Breast, 31 & $1 ; 1$ & $0 ; 1$ \\
\hline $\begin{array}{l}\text { c.5158_5159insA } \\
\text { p.Ser1720Tyrfs*7 }\end{array}$ & $B R C A 2$ & 1 & Frameshift & Breast, 51 & 1 & 1 \\
\hline c.5216dupA p.Tyr1739Ter & $B R C A 2$ & 1 & Frameshift & Bresat, 36 & 3 & 0 \\
\hline c.5682C $>$ G p.Tyr 1894 Ter & $B R C A 2$ & 1 & Nonsense & Breast, 44 & 6 & 0 \\
\hline c.5857G $>$ T p.Glu1953Ter & $B R C A 2$ & 1 & Nonsense & Ovarian, 56 & 3 & 1 \\
\hline $\begin{array}{l}\text { c.6405_6409delCTTAA } \\
\text { p.Asn2135Lysfs*2 }\end{array}$ & $B R C A 2$ & 2 & Frameshift & Breast 41; Breast, 48 & $7 ; 4$ & $0 ; 0$ \\
\hline $\begin{array}{l}\text { c.6611delC } \\
\text { p.Pro2204Leufs*2 }\end{array}$ & $B R C A 2$ & 1 & Frameshift & Breast, 46 & 3 & 0 \\
\hline c.7180A > T p.Arg2394Ter & $B R C A 2$ & 1 & Nonsense & Breast, 34 & 1 & 0 \\
\hline $\begin{array}{l}\text { c. } 7987 \text { delG } \\
\text { p.Glu2663Lysfs*10 }\end{array}$ & $B R C A 2$ & 1 & Frameshift & Breast, 31 & 7 & 0 \\
\hline c. $8023 \mathrm{~A}>\mathrm{G}$ p.Ile $2675 \mathrm{Val}$ & $B R C A 2$ & 1 & Missense & Ovarian, 59 & 3 & 0 \\
\hline c. $8488-1 \mathrm{G}>\mathrm{A}$ & $B R C A 2$ & 1 & Splicing & Breast, 26 & 1 & 0 \\
\hline $\begin{array}{l}\text { c.8711delT } \\
\text { p.Leu2904Leufs*4 }\end{array}$ & $B R C A 2$ & 1 & Frameshift & Breast, 30 & 4 & 1 \\
\hline c. $9382 \mathrm{C}>\mathrm{T}$ p.Arg3128Ter & $B R C A 2$ & 1 & Nonsense & Breast, 53 & 3 & 1 \\
\hline $\begin{array}{l}\text { c. } 9401 \mathrm{delG} \\
\text { p.Gly3134Alafs*29 }\end{array}$ & $B R C A 2$ & 1 & Frameshift & Breast, 35 & 5 & 0 \\
\hline
\end{tabular}

Bold indicates new mutations identified in the present study.

*cases where more the one family carries the same mutation are separated by ";".

identified in patients carrying pathogenic mutations. The variants c. $811 \mathrm{G}>\mathrm{A}(B R C A 1)$ and c. $8187 \mathrm{G}>\mathrm{T}(B R C A 2)$ were identified in a patient carrying the pathogenic mutation c.8023A $>\mathrm{G}$ (p.Ile2675 Val) at the $B R C A 2$ gene. In addition, the alteration c. $8351 \mathrm{G}>\mathrm{A}(B R C A 2)$ was identified in two patients and one of them is carrier of the mutation p.Arg337His at TP53 gene (this patient also has the variant c. $7469 \mathrm{~T}>\mathrm{C}$, at $B R C A 2$ gene). Lastly, the alteration c.7448G $>$ A (BRCA2) was identified in a patient carrying the BRCA1 mutation c.1687C $>\mathrm{T}$ (p.Gln563Ter).

Furthermore, three patients harbor more than one variant. One of them is carrier of the c.7469T $>C$
(BRCA2) and c.8351G $>\mathrm{A}($ BRCA2) variants (this patient also have the pathogenic mutation p.Arg337His at TP53 gene as described above); the other have the alterations c. $4928 \mathrm{~T}>\mathrm{C}$ (BRCA2), and c.8351G $>\mathrm{A}$ (BRCA2). In addition one patient (with $\mathrm{OC}$ at 59 years of age and carrier of the pathogenic mutation c.8023A $>\mathrm{G}$ (p.Ile2675Val) at the $B R C A 2$ gene) has two variants: c. $811 \mathrm{G}>\mathrm{A}(B R C A 1)$ and c. $8187 \mathrm{G}>\mathrm{T}(B R C A 2)$.

The identification of two families with possible pathogenic variants (C65 score) in BRCAl (c.190T $>$ C, p.Cys64Arg, c.5509T $>$ C and p.Trp1837Arg) as well as three families carrying variants with the same score 
in $B R C A 2$ (c.4585G $>$ A, p.Gly1529Arg; c.6935A $>$ T, p.Asp2312Val, c.7994A $>\mathrm{G}$ and p.Asp2665Gly) was possible using the AlignGVGD program. The probands of four out of these 5 families have had BC at very early ages (ages between 27 and 30 years as can be seen in details at Table 3 ). The only family with tumors at more advanced ages among those with variants classified as C65 is the family with the variant c.7994A $>\mathrm{G}$ at $B R C A 2$ gene, where the proband had $\mathrm{BC}$ at 60 years old. However, this woman has two relatives with BC (at 35 and 66 years respectively) and one with OC (age at diagnosis unknown).

An alteration located in a splicing region in the $B R C A 1$ gene (variant c.5153-2A $>$ C) was also identified in a patient with $\mathrm{BC}$ at 28 years old. However, since there was no previous report in the literature, as well as no functional study performed supporting their nonfunctionality, this variant was not considered as proven pathogenic. Similarly, the BRCA2 variants (missense) c.6554C $>$ T (p.Ala2185Val), c.6988A $>$ G (p.Ile2330Val) and c. $8755 \mathrm{G}>\mathrm{T}$ (p.Gly2919Cys) have not been previously described in the literature and given the lack of information about their biological impact they have been "provisionally" considered VUS. However it is important to highlight that the patient with the variant c.6988A $>\mathrm{G}$ $(B R C A 2)$ had a personal history of $\mathrm{BBC}$ at 24 years of age and 2 relatives with $\mathrm{BC}$, raising strong suspicion about its pathogenicity (although classified as benign by the in silico tools used).

Two other identified variants that call our attention were: (i) the c.5096G $>\mathrm{A}$ (BRCA1), identified in a family without $\mathrm{BC}$ history (proband with $\mathrm{OC}$ at 44 and one relative with $\mathrm{OC}$ ), which was the only variant identified in families with cancer history exclusive of OC and (ii) the in frame deletion c.5425_5430delGTTGTG, due to the severity of the family history (proband with BC at 36 years old and 7 cases of $\mathrm{BC}$ among first, second and third degree relatives). In the same way, two families with the alteration c.6347A $>\mathrm{G}(B R C A 2)$ had probands with $\mathrm{BC}$ at 38 and 26 years respectively, and 4 cases of $\mathrm{BC}$ in each family were reported.

For the 274 index cases with no identified BRCA1/ $B R C A 2$ germline mutation, test for the TP53 gene was performed, which detected pathogenic mutation in 9 patients (data not shown). All these 9 families had probands with $\mathrm{BC}$ diagnosed before the age of 50 (varying from 27 to 48 years) and at least one first or second degree relative with $\mathrm{BC}$. All 9 families are carriers of the Brazilian founder mutation p.Arg337His (exon 10 of TP53 gene) [21].

\section{Ancestry}

We further performed genetic ancestry assessment on 341 of the 349 participants (Figure 2) using 46 ancestry-informative markers. Although overall ancestry estimates computed with Admixture and Structure algorithms were similar, for simplicity we will refer only to the former one. Average ancestry proportions in our group were 70.6\% European, 14.5\% African, 8.0\% Native American and 6.8\% East Asian.

There was no statistically significant difference in the ancestry profile of WT individuals when compared to those with germline mutation in $B R C A 1 / B R C A 2$ genes (Supplementary Table S2 and Figure 2). Additionally, there is no difference in the ancestry profile between $B R C A 1$ and BRCA2 mutated women $(p=0.434)$.

To verify the association of ancestry with clinical characteristics, patients were subdivided according to the BRCA1/BRCA2 status: WT or mutated. Among the mutated ones (BRCA1 or BRCA2), no association with clinico-pathological characteristics and genetic ancestry was observed.

On the other hand, we observed that the African ancestry component among individuals without BRCA1/ $B R C A 2$ mutations was associated with higher tumor

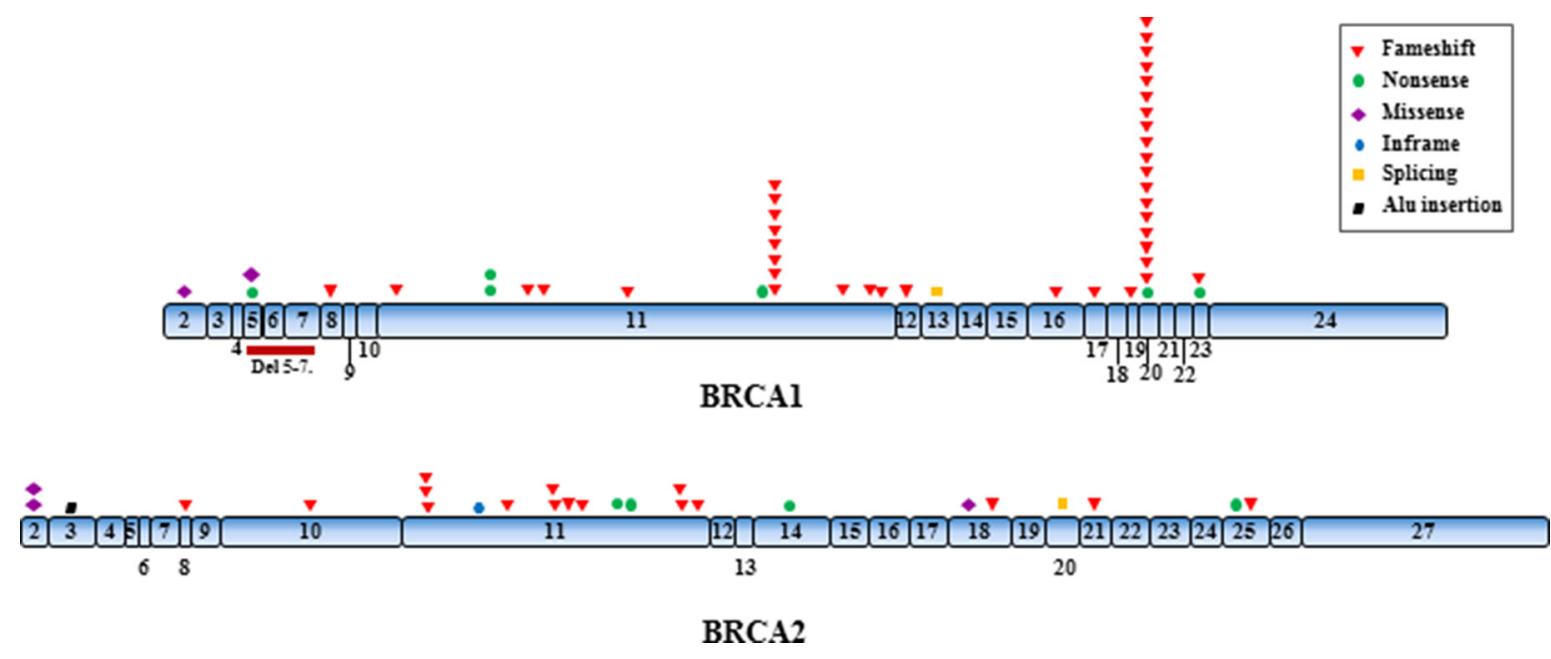

Figure 1: Distribution of the identified mutations along the $B R C A 1$ and $B R C A 2$ genes. 
Table 2: Variants of unknown significance according to 7 databases an 5 in silico prediction programs

\begin{tabular}{|c|c|c|c|c|c|c|c|c|c|c|c|c|}
\hline & HGMD & BIC & LOVD & $\begin{array}{l}\text { LOVD } \\
\text { IARC }\end{array}$ & ClinVar & ARUP & $\begin{array}{c}\text { BRCA } \\
\text { Share }^{\mathrm{TM}}\end{array}$ & $\begin{array}{l}\text { Polyphen- } \\
2\end{array}$ & SIFT & $\begin{array}{l}\text { Align- } \\
\text { GVGD }\end{array}$ & MAPP & CADD \\
\hline \multicolumn{13}{|l|}{$B R C A 1$} \\
\hline c.190T>C; p.Cys64Arg & Yes & VUS & $\begin{array}{l}\text { Normal RNA } \\
\text { splicing }\end{array}$ & $N / R$ & $\begin{array}{c}\text { conflicting } \\
\text { data }\end{array}$ & $\mathrm{N} / \mathrm{R}$ & Causal & $\begin{array}{l}\text { probably } \\
\text { damaging }\end{array}$ & $\begin{array}{l}\text { Predict Not } \\
\text { Tolerated }\end{array}$ & C65 & Not tolerated & NC \\
\hline c. $811 \mathrm{G}>\mathrm{A} ;$ p.Val271Met & Yes & VUS & $\begin{array}{l}\text { probably } \\
\text { neutral }\end{array}$ & $\mathrm{N} / \mathrm{R}$ & VUS & $\mathrm{N} / \mathrm{R}$ & $\mathrm{N} / \mathrm{R}$ & $\begin{array}{c}\text { possibly } \\
\text { damaging }\end{array}$ & Predict Tolerated & $\mathrm{C} 0$ & Not tolerated & $\mathrm{NC}$ \\
\hline c. $1601 \mathrm{~A}>\mathrm{G} ; \mathrm{p} . \mathrm{G} \ln 534 \mathrm{Arg}$ & $\mathrm{N} / \mathrm{R}$ & vUS & $\mathrm{N} / \mathrm{R}$ & $N / R$ & VUS & $\mathrm{N} / \mathrm{R}$ & VUS & benign & $\begin{array}{l}\text { Predict Not } \\
\text { Tolerated }\end{array}$ & $\mathrm{C} 0$ & Not tolerated & $\begin{array}{l}\text { High } \\
\text { risk }\end{array}$ \\
\hline c. $1648 \mathrm{~A}>$ C p.Asn 550 His & Yes & VUS & $\begin{array}{c}\text { probably } \\
\text { neutral }\end{array}$ & class 1 & $\begin{array}{c}\text { conflicting } \\
\text { data }\end{array}$ & Class 1 & Neutral & $\begin{array}{l}\text { probably } \\
\text { damaging }\end{array}$ & $\begin{array}{l}\text { Predict Not } \\
\text { Tolerated }\end{array}$ & $\mathrm{C} 0$ & Tolerated & NC \\
\hline c. $3823 \mathrm{~A}>\mathrm{G}$ p.Ile $1275 \mathrm{Val}$ & $\mathrm{N} / \mathrm{R}$ & VUS & $\begin{array}{l}\text { probably } \\
\text { neutral }\end{array}$ & $N / R$ & $\begin{array}{c}\text { conflicting } \\
\text { data }\end{array}$ & $\mathrm{N} / \mathrm{R}$ & Neutral & benign & Predict Tolerated & $\mathrm{C} 0$ & Tolerated & $\mathrm{NC}$ \\
\hline c. $4484 \mathrm{G}>$ A p.Arg 1495 Lys & VUS & yes & $\mathrm{N} / \mathrm{R}$ & $\mathrm{N} / \mathrm{R}$ & $\begin{array}{l}\text { conflicting } \\
\text { data }\end{array}$ & $\mathrm{N} / \mathrm{R}$ & Causal & benign & $\begin{array}{l}\text { Predict Not } \\
\text { Tolerated }\end{array}$ & $\mathrm{C} 0$ & Not tolerated & NC \\
\hline $\begin{array}{l}\text { c.5062_5064delGTT; } \\
\text { p.Val1688del }\end{array}$ & Yes & VUS & $\begin{array}{l}\text { No splicing } \\
\text { defect }\end{array}$ & $N / R$ & $\begin{array}{c}\text { conflicting } \\
\text { data }\end{array}$ & Class 5 & Causal & $\mathrm{NC}$ & NCR & $\mathrm{NC}$ & NC & NC \\
\hline c. $5096 \mathrm{G}>\mathrm{A}$ p.Arg $1699 \mathrm{Gln}$ & Yes & VUS & $\begin{array}{l}\text { probably } \\
\text { deleterious }\end{array}$ & $\mathrm{N} / \mathrm{R}$ & $\begin{array}{c}\text { conflicting } \\
\text { data }\end{array}$ & $\mathrm{N} / \mathrm{R}$ & VUS & $\begin{array}{l}\text { probably } \\
\text { damaging }\end{array}$ & $\begin{array}{l}\text { Predict Not } \\
\text { Tolerated }\end{array}$ & $\mathrm{C} 35$ & Not tolerated & $\begin{array}{l}\text { Medium } \\
\text { risk }\end{array}$ \\
\hline c. $5153-2 \mathrm{~A}>\mathrm{C}$ & $\mathrm{N} / \mathrm{R}$ & $\mathrm{N} / \mathrm{R}$ & $\mathrm{N} / \mathrm{R}$ & $N / R$ & $\mathrm{~N} / \mathrm{R}$ & $\mathrm{N} / \mathrm{R}$ & $\mathrm{N} / \mathrm{R}$ & $\mathrm{NC}$ & $\mathrm{NC}$ & $\mathrm{NC}$ & $\mathrm{NC}$ & $\mathrm{NC}$ \\
\hline \multicolumn{13}{|l|}{ c.5425_5430delGTTGTG } \\
\hline & $\mathrm{N} / \mathrm{R}$ & vUS & $\mathrm{N} / \mathrm{R}$ & $\mathrm{N} / \mathrm{R}$ & VUS & $\mathrm{N} / \mathrm{R}$ & $N / R$ & $\mathrm{NC}$ & $\mathrm{NC}$ & $\mathrm{NC}$ & $\mathrm{NC}$ & NC \\
\hline c.5509T >C; p.Trp1837Arg & Yes & VUS & $\begin{array}{l}\text { probably } \\
\text { deleterious }\end{array}$ & $\mathrm{N} / \mathrm{R}$ & $\begin{array}{l}\text { conflicting } \\
\text { data }\end{array}$ & $\mathrm{N} / \mathrm{R}$ & VUS & $\begin{array}{l}\text { probably } \\
\text { damaging }\end{array}$ & $\begin{array}{l}\text { Predict Not } \\
\text { Tolerated }\end{array}$ & C65 & Not tolerated & NC \\
\hline \multicolumn{13}{|l|}{ BRCA2 } \\
\hline c. $223 \mathrm{G}>$ C p.Ala75Pro & Yes & vUS & $\begin{array}{l}\text { probably } \\
\text { neutral }\end{array}$ & class 1 & $\begin{array}{c}\text { conflicting } \\
\text { data }\end{array}$ & Class 1 & Neutral & $\begin{array}{l}\text { probably } \\
\text { damaging }\end{array}$ & $\begin{array}{l}\text { Predict Not } \\
\text { Tolerated }\end{array}$ & $\mathrm{C} 0$ & Not tolerated & NC \\
\hline c. $1798 \mathrm{~T}>\mathrm{C}$ p.Tyr600His & $\mathrm{N} / \mathrm{R}$ & vUS & $\mathrm{N} / \mathrm{R}$ & $\mathrm{N} / \mathrm{R}$ & $\begin{array}{c}\text { conflicting } \\
\text { data }\end{array}$ & $\mathrm{N} / \mathrm{R}$ & VUS & benign & Predict Tolerated & $\mathrm{C} 0$ & Tolerated & $\mathrm{NC}$ \\
\hline c. $2274 \mathrm{~T}>$ G p.Ser758Arg & $\mathrm{N} / \mathrm{R}$ & $\mathrm{N} / \mathrm{R}$ & $N / R$ & $\mathrm{~N} / \mathrm{R}$ & VUS & $\mathrm{N} / \mathrm{R}$ & $\mathrm{N} / \mathrm{R}$ & benign & Predict Tolerated & $\mathrm{C} 0$ & Not tolerated & $\begin{array}{l}\text { Low } \\
\text { risk }\end{array}$ \\
\hline c. $2350 \mathrm{~A}>\mathrm{G}$ p.Met $784 \mathrm{His}$ & VUS & VUS & $\begin{array}{c}\text { probably } \\
\text { neutral }\end{array}$ & class 3 & $\mathrm{~N} / \mathrm{R}$ & Class 3 & VUS & $\begin{array}{l}\text { possibly } \\
\text { damaging }\end{array}$ & $\begin{array}{l}\text { Predict Not } \\
\text { Tolerated }\end{array}$ & $\mathrm{C} 0$ & Not tolerated & $\mathrm{NC}$ \\
\hline c. $2503 \mathrm{C}>\mathrm{T}$ p.Pro835Ser & $\mathrm{N} / \mathrm{R}$ & VUS & $\mathrm{N} / \mathrm{R}$ & $N / R$ & VUS & $\mathrm{N} / \mathrm{R}$ & $N / R$ & benign & Predict Tolerated & $\mathrm{C} 0$ & Tolerated & $\begin{array}{l}\text { Low } \\
\text { risk }\end{array}$ \\
\hline c. $4585 \mathrm{G}>$ A p.Gly $1529 \mathrm{Arg}$ & Yes & no & inconclusive & class 1 & benign & Class 1 & Neutral & $\begin{array}{l}\text { probably } \\
\text { damaging }\end{array}$ & $\begin{array}{l}\text { Predict Not } \\
\text { Tolerated }\end{array}$ & C65 & Tolerated & NC \\
\hline c. $4681 \mathrm{C}>$ A p.His 1561 Asn & $\mathrm{N} / \mathrm{R}$ & VUS & $\mathrm{N} / \mathrm{R}$ & $\mathrm{N} / \mathrm{R}$ & $\begin{array}{c}\text { conflicting } \\
\text { data }\end{array}$ & $\mathrm{N} / \mathrm{R}$ & VUS & benign & Predict Tolerated & $\mathrm{C} 0$ & Tolerated & $\mathrm{NC}$ \\
\hline c. $4928 \mathrm{~T}>$ C p.Val1643Ala & $\mathrm{N} / \mathrm{R}$ & VUS & inconclusive & class 3 & $\begin{array}{c}\text { conflicting } \\
\text { data }\end{array}$ & Class 3 & VUS & benign & Predict Tolerated & $\mathrm{C} 0$ & Tolerated & $\mathrm{NC}$ \\
\hline c. $5096 \mathrm{~A}>$ G p.Asp 1699 Gly & $\mathrm{N} / \mathrm{R}$ & VUS & $\mathrm{N} / \mathrm{R}$ & $N / R$ & VUS & $\mathrm{N} / \mathrm{R}$ & $\mathrm{N} / \mathrm{R}$ & benign & Predict Tolerated & $\mathrm{C} 0$ & Not tolerated & NC \\
\hline c. $5640 \mathrm{~T}>$ G p.Asn 1880 Lys & Yes & VUS & $\begin{array}{l}\text { probably } \\
\text { neutral }\end{array}$ & $\mathrm{N} / \mathrm{R}$ & $\begin{array}{c}\text { conflicting } \\
\text { data }\end{array}$ & $\mathrm{N} / \mathrm{R}$ & Polymorphism & benign & $\begin{array}{l}\text { Predict Not } \\
\text { Tolerated }\end{array}$ & $\mathrm{C} 0$ & Not tolerated & $\mathrm{NC}$ \\
\hline c. $6347 \mathrm{~A}>\mathrm{G}$ p.His2116Arg & Yes & no & $\mathrm{N} / \mathrm{R}$ & $\mathrm{N} / \mathrm{R}$ & benign & $\mathrm{N} / \mathrm{R}$ & Neutral & $\begin{array}{c}\text { possibly } \\
\text { damaging }\end{array}$ & $\begin{array}{l}\text { Predict Not } \\
\text { Tolerated }\end{array}$ & $\mathrm{C} 0$ & Tolerated & $\mathrm{NC}$ \\
\hline c. $6412 \mathrm{G}>\mathrm{T}$ p.Val2138Phe & $\mathrm{N} / \mathrm{R}$ & VUS & $\begin{array}{l}\text { probably } \\
\text { neutral }\end{array}$ & $\mathrm{N} / \mathrm{R}$ & $\begin{array}{c}\text { conflicting } \\
\text { data }\end{array}$ & $\mathrm{N} / \mathrm{R}$ & VUS & benign & $\begin{array}{l}\text { Predict Not } \\
\text { Tolerated }\end{array}$ & $\mathrm{C} 0$ & Tolerated & $\mathrm{NC}$ \\
\hline c. $6554 \mathrm{C}>\mathrm{T}$ p.Ala2185 Val & $\mathrm{N} / \mathrm{R}$ & $\mathrm{N} / \mathrm{R}$ & $\mathrm{N} / \mathrm{R}$ & $\mathrm{N} / \mathrm{R}$ & $\mathrm{N} / \mathrm{R}$ & $\mathrm{N} / \mathrm{R}$ & $\mathrm{N} / \mathrm{R}$ & benign & Predict Tolerated & $\mathrm{C} 0$ & Tolerated & $\begin{array}{l}\text { Low } \\
\text { risk }\end{array}$ \\
\hline c. $6935 \mathrm{~A}>\mathrm{T}$ p.Asp $2312 \mathrm{Val}$ & VUS & VUS & $\begin{array}{l}\text { probably } \\
\text { neutral }\end{array}$ & $\mathrm{N} / \mathrm{R}$ & VUS & $\mathrm{N} / \mathrm{R}$ & VUS & $\begin{array}{l}\text { probably } \\
\text { damaging }\end{array}$ & $\begin{array}{l}\text { Predict Not } \\
\text { Tolerated }\end{array}$ & C65 & Not tolerated & NC \\
\hline c. $6988 \mathrm{~A}>\mathrm{G}$ p.Ile2330Val & $\mathrm{N} / \mathrm{R}$ & $\mathrm{N} / \mathrm{R}$ & $\mathrm{N} / \mathrm{R}$ & $\mathrm{N} / \mathrm{R}$ & $\mathrm{N} / \mathrm{R}$ & $\mathrm{N} / \mathrm{R}$ & $\mathrm{N} / \mathrm{R}$ & benign & Predict Tolerated & $\mathrm{C} 0$ & Not tolerated & $\mathrm{NC}$ \\
\hline c. $7017 \mathrm{G}>$ C p.Lys2339Asn & VUS & no & $\mathrm{N} / \mathrm{R}$ & $\mathrm{N} / \mathrm{R}$ & $\begin{array}{c}\text { conflicting } \\
\text { data }\end{array}$ & $\mathrm{N} / \mathrm{R}$ & Likely neutral & benign & Predict Tolerated & $\mathrm{C} 0$ & Not tolerated & $\mathrm{NC}$ \\
\hline c. $7448 \mathrm{G}>$ A p.Ser2483Asn & $\mathrm{N} / \mathrm{R}$ & VUS & $\begin{array}{c}\text { probably } \\
\text { neutral }\end{array}$ & $\mathrm{N} / \mathrm{R}$ & $\begin{array}{c}\text { conflicting } \\
\text { data }\end{array}$ & $\mathrm{N} / \mathrm{R}$ & VUS & benign & Predict Tolerated & $\mathrm{C} 0$ & Tolerated & $\begin{array}{l}\text { Low } \\
\text { risk }\end{array}$ \\
\hline c. $7469 \mathrm{~T}>\mathrm{C}$ p.Ile2490Thr & VUS & no & $\begin{array}{l}\text { probably } \\
\text { neutral }\end{array}$ & $\mathrm{N} / \mathrm{R}$ & $\begin{array}{c}\text { conflicting } \\
\text { data }\end{array}$ & $\mathrm{N} / \mathrm{R}$ & VUS & benign & Predict Tolerated & $\mathrm{C} 45$ & Not tolerated & $\mathrm{NC}$ \\
\hline c. $7507 \mathrm{G}>$ A p. Val2503Ile & $\mathrm{N} / \mathrm{R}$ & $\mathrm{N} / \mathrm{R}$ & $\mathrm{N} / \mathrm{R}$ & $\mathrm{N} / \mathrm{R}$ & VUS & $\mathrm{N} / \mathrm{R}$ & VUS & benign & Predict Tolerated & $\mathrm{C} 0$ & Tolerated & $\begin{array}{l}\text { Low } \\
\text { risk }\end{array}$ \\
\hline
\end{tabular}




\begin{tabular}{|c|c|c|c|c|c|c|c|c|c|c|c|c|}
\hline c.7994A >G p.Asp2665Gly & VUS & no & $\begin{array}{c}\text { probably } \\
\text { neutral }\end{array}$ & class 2 & bening & Class 2 & VUS & $\begin{array}{c}\text { probably } \\
\text { damaging }\end{array}$ & $\begin{array}{l}\text { Predict Not } \\
\text { Tolerated }\end{array}$ & $\mathrm{C} 65$ & Not tolerated & $\begin{array}{l}\text { Low } \\
\text { risk }\end{array}$ \\
\hline c. $8187 \mathrm{G}>$ T P.Lys2729Asn & VUS & VUS & $\begin{array}{c}\text { probably } \\
\text { neutral }\end{array}$ & class 1 & $\begin{array}{c}\text { conflicting } \\
\text { data }\end{array}$ & Class 1 & VUS & $\begin{array}{l}\text { probably } \\
\text { damaging }\end{array}$ & $\begin{array}{c}\text { Predict Not } \\
\text { Tolerated }\end{array}$ & $\mathrm{C} 35$ & Not tolerated & $\begin{array}{l}\text { Low } \\
\text { risk }\end{array}$ \\
\hline c. $8351 \mathrm{G}>$ A p.Arg2784Gln & Yes & VUS & $\begin{array}{c}\text { probably } \\
\text { deleterious }\end{array}$ & $\mathrm{N} / \mathrm{R}$ & VUS & $\mathrm{N} / \mathrm{R}$ & VUS & $\begin{array}{l}\text { probably } \\
\text { damaging }\end{array}$ & $\begin{array}{c}\text { Predict Not } \\
\text { Tolerated }\end{array}$ & $\mathrm{C} 35$ & Not tolerated & $\mathrm{NC}$ \\
\hline c. $8755 \mathrm{G}>$ T p.Gly 2919 Cys & $\mathrm{N} / \mathrm{R}$ & $\mathrm{N} / \mathrm{R}$ & $\mathrm{N} / \mathrm{R}$ & $\mathrm{N} / \mathrm{R}$ & $\mathrm{N} / \mathrm{R}$ & $\mathrm{N} / \mathrm{R}$ & $\mathrm{N} / \mathrm{R}$ & $\begin{array}{c}\text { probably } \\
\text { damaging }\end{array}$ & $\begin{array}{c}\text { Predict Not } \\
\text { Tolerated }\end{array}$ & $\mathrm{C} 15$ & Tolerated & $\begin{array}{l}\text { Low } \\
\text { risk }\end{array}$ \\
\hline c. $9235 \mathrm{G}>$ A p.Val3079Ile & $\mathrm{N} / \mathrm{R}$ & VUS & $\begin{array}{c}\text { probably } \\
\text { neutral }\end{array}$ & class 1 & $\begin{array}{c}\text { conflicting } \\
\text { data }\end{array}$ & Class 1 & VUS & $\begin{array}{c}\text { possibly } \\
\text { damaging }\end{array}$ & Predict Tolerated & $\mathrm{C} 0$ & Tolerated & $\mathrm{NC}$ \\
\hline c. $9730 \mathrm{G}>$ A p.Val3244Ile & $\mathrm{N} / \mathrm{R}$ & no & $\mathrm{N} / \mathrm{R}$ & $\mathrm{N} / \mathrm{R}$ & $\begin{array}{c}\text { conflicting } \\
\text { data }\end{array}$ & $\mathrm{N} / \mathrm{R}$ & VUS & Benign & Predict Tolerated & $\mathrm{C} 0$ & Tolerated & $\mathrm{NC}$ \\
\hline
\end{tabular}

NR: not reported; NC: not calculated; VUS: variant of unknown significance.

Align-GVGD scores: C0: neutral, C15: low risk of protein function alteration, $\mathrm{C} 25$ : low to medium risk of protein function alteration, C35: medium to high risk of protein function alteration, C45: high risk of protein function alteration, C55: high risk of a pathogenic protein function alteration, C65: pathogenic protein function alteration.

Table 3: Information regarding tumor history, frequency and segregation of the variants of unknown significance

\begin{tabular}{|c|c|c|c|}
\hline NOF $\begin{array}{c}\text { Co- } \\
\text { segregation }\end{array}$ & $\begin{array}{c}\text { Tumors in index patient, } \\
\text { age at onset** }\end{array}$ & $\begin{array}{l}\text { Number of } \\
\text { BC cases in } \\
\text { relatives* }\end{array}$ & $\begin{array}{c}\text { Number of OC } \\
\text { cases in relatives* }\end{array}$ \\
\hline
\end{tabular}

BRCA1

c.811G $>$ A; p.Val271Met 1 yes

Breast, 30

0

c.1601A $>$ G; p.Gln534Arg 1 no

Ovarian, 59

2

Breast, 51

c. $1648 \mathrm{~A}>$ C p.Asn550His 1 no

Breast, 49

c.3823A $>$ G p.Ile1275Val 1 no

Breast, 43

c.4484G $>$ A p.Arg1495Lys 1 no

Breast, 26

c.5062_5064delGTT;

Breast, 33

Ovarian, 44

Breast, 28

Breast, 36

Breast, 28

Breast, 20; Breast, 54

Ovarian, 46

Breast, 35

Breast, 55

Breast, 54

Breast, 27

Breast, 28

Breast, 40

Breast, 24

c.5096A $>$ G p.Asp1699Gly 1

c.5640T $>$ G p.Asn1880Lys 2 no

c.6347A $>$ G p.His2116Arg 3 no

Breast, 27; Breast, 36

Breast, 38; Breast, 26;

Breast, 44

c.6412G $>$ T p.Val2138Phe 1 no Breast, 49

Breast, 31

$\begin{array}{ll}3 & 1 \\ 5 & 0 \\ 4 & 0 \\ 1 & 0 \\ 3 & 0 \\ 2 & 0 \\ 0 & \\ 1 & 1 \\ 7 & 0 \\ 0 & 0\end{array}$

c.6554C $>$ T p.Ala2185Val 1 no

$\begin{array}{cc}1 ; 3 & 0 ; 0 \\ 0 & 0 \\ 2 & 0 \\ 3 & 0 \\ 2 & 0 \\ 2 & 0 \\ 1 & 0 \\ 2 & 0 \\ 1 & 0 \\ 1 ; 2 & 0 ; 0 \\ 4 ; 1 ; 4 & 0 ; 0 ; ? \\ 2 & 0 \\ 2 & 0\end{array}$




\begin{tabular}{|c|c|c|c|c|c|}
\hline c. $6935 \mathrm{~A}>$ T p.Asp2312Val & 1 & no & Breast, 28 & 1 & 0 \\
\hline c.6988A $>$ G p.Ile2330Val & 1 & no & Breast, 24 & 2 & 0 \\
\hline c.7017G $>$ C p.Lys2339Asn & 2 & no & Ovarian, 33; Breast, 74 & $2 ; 1$ & $1 ; 0$ \\
\hline c. $7448 \mathrm{G}>$ A p.Ser2483Asn & 1 & yes & Breast, 29 & 4 & 0 \\
\hline c.7469T $>$ C p.Ile2490Thr & 10 & yes & $\begin{array}{l}\text { Breast, 29; Breast, 39; } \\
\text { Breast, 29; Breast, 38; } \\
\text { Breast, 43; Breast, 40; } \\
\text { Breast, 37; Breast, 41; } \\
\text { Bresat, 61; Breast, 29; }\end{array}$ & $1 ; 3 ; 1 ; 1 ; 4 ; 2 ; 6 ; 1 ; 3 ; 1$ & $0 ; 0 ; 0 ; 0 ; 0 ; 0 ; 0 ; 0 ; 0 ; 0$ \\
\hline c.7507G $>$ A p.Val2503Ile & 1 & no & Breast, 37 & 2 & 0 \\
\hline c.7994A>G p.Asp2665Gly & 1 & no & Breast, 60 & 2 & 1 \\
\hline c. $8187 \mathrm{G}>$ T P.Lys2729Asn & 1 & yes & Ovarian, 59 & 3 & 0 \\
\hline c. $8351 \mathrm{G}>$ A p.Arg2784Gln & 2 & yes & Breast, 41; Breast, 40 & $1 ; 2$ & $0 ; 0$ \\
\hline c. $8755 \mathrm{G}>$ T p.Gly2919Cys & 1 & no & Breast, 32 & 0 & 0 \\
\hline c.9235G>A p.Val3079Ile & 1 & no & Breast, 55 & 2 & 0 \\
\hline c. $9730 \mathrm{G}>$ A p. Val3244Ile & 2 & no & Ovarian, 33; Breast, 74 & $2 ; 1$ & $1 ; 0$ \\
\hline
\end{tabular}

*cases where more the one family carries the same mutation are separated by “;”.

grade $(p=0.008)$. Furthermore, we found out that when the African component increases in an individual, the probability of $\mathrm{BBC}$ decreases $(p=0.005)$ and the number of cancer cases in the family increases $(p=0.04)$.

In 15 out of 18 patients with c.5266dupC mutation in the BRCAl gene, the European ancestry component was predominant (over 69\%) (Figure 3). There was no statistically significant difference in the ancestry component of patients carrying the c.5266dupC mutation vs. those with other $B R C A 1 / B R C A 2$ mutations. For one patient, there was a similar balance between the Amerindian (0.482) and European (0.478) components (Figure 3). In another family, there was a similar distribution among three components: 0.387 for the European, 0.280 for the Amerindian and 0.261 for the African component (Figure 3).

\section{DISCUSSION}

The present work is the largest study analyzing the $B R C A 1 / B R C A 2$ germline mutation frequencies in the Brazilian population, with a prevalence of $21.5 \%$ of deleterious mutations, being $13.6 \%$ new mutations (not described in the literature) and $88.6 \%$ of them never described in the Brazilian population. Although it represents the data from a single Institution, the $\mathrm{BCH}$ receives patients from all over Brazil. In this particular study, the majority $(71.6 \%)$ came from the Southeast region of Brazil, followed by the Central-West (18.3\%), North (7.2\%), and the Northeast and South regions (1.4\% each), covering 16 Brazilian States. Moreover, given the highly admixed Brazilian population, and the potential impact of the presence of founder mutations, this study presents a characterization of the genetic ancestry of this cohort.

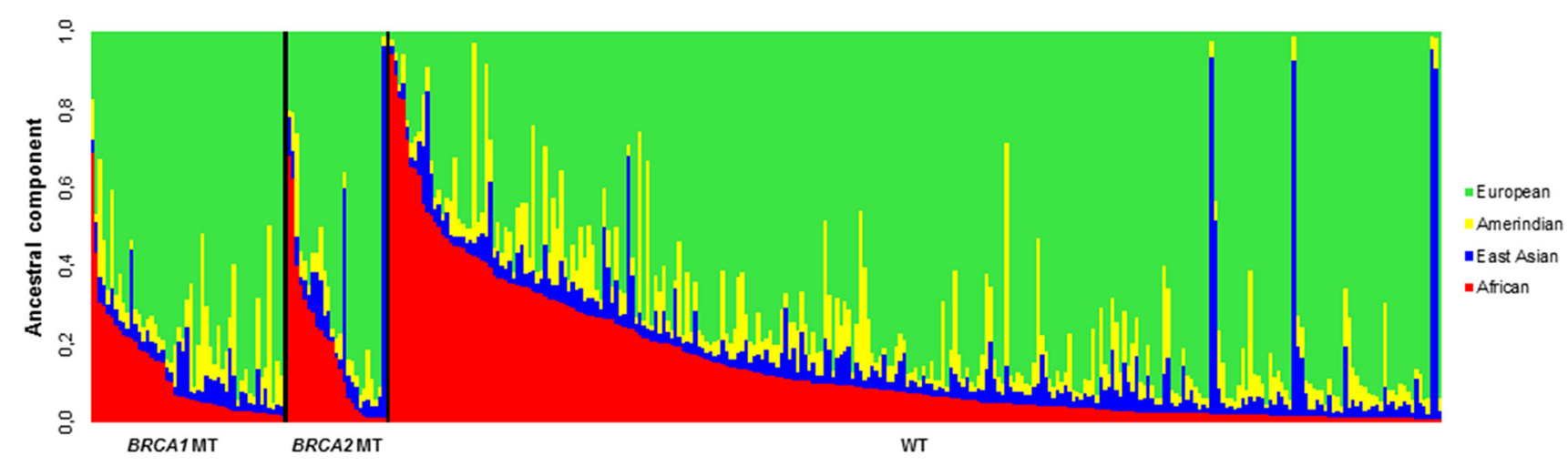

Figure 2: Ancestral profile of the studied patient group showing individual ancestry estimates according to mutational status. $B R C A 1$ mutated (left), BRCA2 mutated (centre) and BRCA1/BRCA2 WT (right). 
We observed a frequency of $21.5 \% \mathrm{BRCA1/BRCA2}$ mutated patients, with most of the mutations located in the $B R C A 1$ gene $(65.3 \%)$. Other than the results reported in this manuscript there are just a few studies on the subject carried out in the Brazilian population [7-13, 22-24] and they are depicted in Table 4.

As shown in Table 4, there are only three studies involving the analysis of the entire coding region of the BRCA1/BRCA2: the study published by Carraro and colleagues in 2013 [24] that examined 54 women with BC diagnosed before the age of 35 , the study published by Silva et al. in 2014 [23] which included 120 women and ours, that analyzed 349 index cases of suspected HBOC families. Although the inclusion criteria may differ, the three studies identified a similar percentage of mutations, $20.4 \%, 22.5 \%$ and $21.5 \%$ respectively. Other studies involving larger sample sizes were performed, however they investigated the presence of specific regions of the $B R C A 1 / B R C A 2$ genes or specific mutations (founder mutations), and the percentage of mutations identified were quite low $[8,11]$.

The fraction of pathogenic mutations identified in our study is in accordance with recent published studies all over the world. A study conducted by Peixoto et al. analyzed the entire $B R C A 1 / B R C A 2$ coding region of 524 Portuguese patients and identified 112/524 families $(21.4 \%)$ with pathogenic mutation [25]. Kang and collaborators analyzed 2403 Korean index patients and found that the prevalence of $B R C A$ mutations in $\mathrm{BC}$ patients with a family history of breast/ovarian cancer was $22.3 \%$ [26]. Churpek and colleagues [27] studied a group of 289 African-American women with family history of $\mathrm{BC}$ and the rate of identified pathogenic mutations was $22 \%$. In a similar way, Weitzel and colleagues [28] evaluated 746 Hispanics with personal and/or family history of breast or OC, and found the presence of BRCA1/ $B R C A 2$ mutations in $25 \%$ of them. In Colombia, a study conducted by Torres and collaborators found a frequency of $24.5 \%$ of deleterious BRCA1/BRCA2 mutations in a cohort of 53 breast/ovarian cancer families evaluated [29]. Finally, data on 10 years of genetic testing (November 1996 to March 2006) conducted by Myriad Laboratory (USA), representing more than $95 \%$ of genetic testing for $B R C A 1 / B R C A 2$ genes performed in the United States, notselected by breast/ovarian cancer family history, points to a frequency of $12.5 \%$ identified deleterious mutations $(5,780$ mutated individuals in a total of 46.276 individuals consecutively tested) [30].

In the present study we found that, among patients with mutated $B R C A 1,77.5 \%$ had a personal history of $\mathrm{BC}$, and $22.4 \%$ of OC. Regarding $B R C A 2,84.6 \%$ had previous personal history of BC and $15.4 \%$ of OC. This is similar to the results recently published by Rebbeck and colleagues [31]. Rebbeck's results show that the proportion of canceraffected individuals carrying mutated BRCA1 with BC was $72.9 \%$, with OC $18.7 \%$ and with a personal history of breast and ovarian cancer was $8.4 \%$. Regarding $B R C A 2$, $86.6 \%$ of the mutated patients had BC, $9.6 \%$ OC and $3.8 \%$ presented previous personal history of both tumors.

We observed that two BRCA1 mutations are responsible for more than $50 \%$ of the mutations identified in this gene in our cohort: the c.5266dupC accounting for $36.7 \%$ of mutations in the BRCA1 gene and for $24 \%$ of all mutations found in this study and the mutation c.3331_3334delCAAG, that represents $16.3 \%$ of our BRCA1 mutations and $10.7 \%$ of the mutations identified. Although the present study is not representative of the Brazilian population diversity, the fact that i) two mutations account for over $50 \%$ of the identified mutations and ii) these two alterations were not concentrated in only one Brazilian region, may serve as a first approach strategy for genetic testing, not only in Brazil, but also in countries/services where the financial resources are limited and where the public health system or private plans do not cover such molecular analysis. If negative results are found

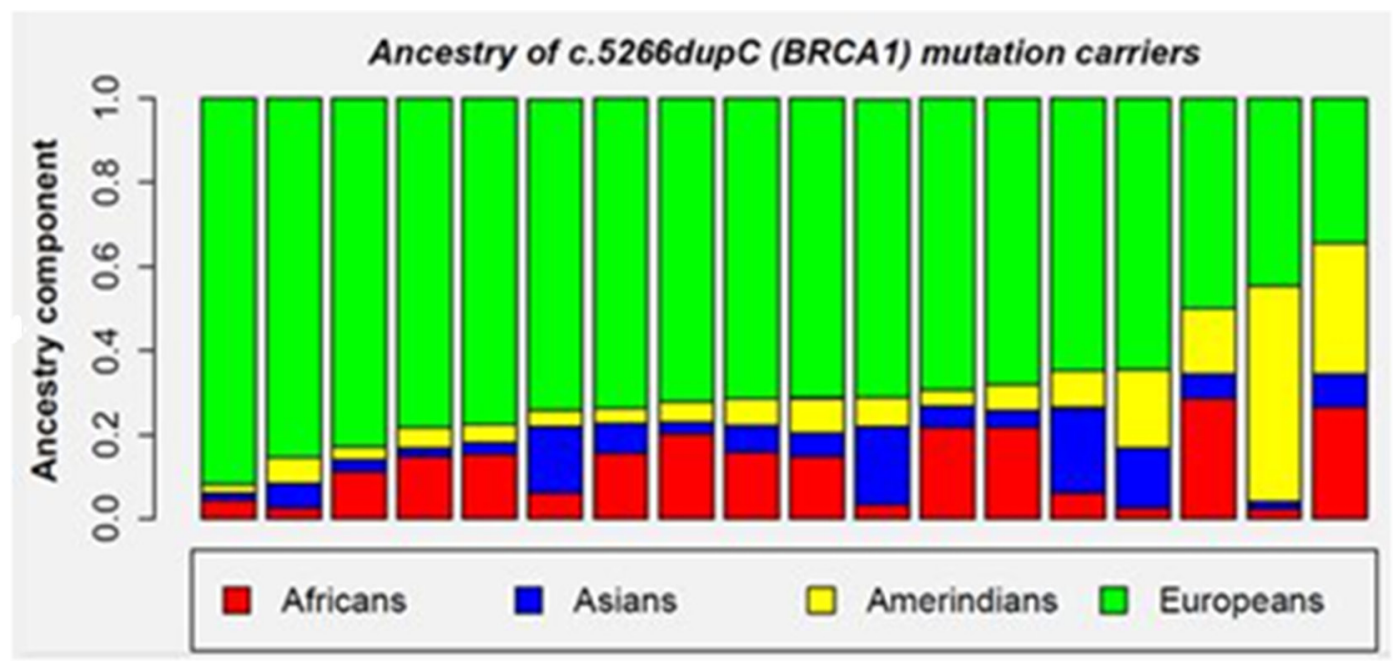

Figure 3: Ancestry contribute on of c.5266dupC mutated patients. 
Table 4: Summary of Brazilian $B R C A 1$ and/or $B R C A 2$ studies

\begin{tabular}{|c|c|c|c|c|}
\hline Sample size & $\begin{array}{c}\% \text { of } B R C A 1 / \\
B R C A 2 \text { mutations }\end{array}$ & Selection criteria & Genetic test performed & Authors, year \\
\hline 47 & $7(14.9 \%)$ & HBOC patients & All coding regions of $B R C A 1$ & Lourenço, 2004 \\
\hline 31 & $4(9.6 \%)$ & HBOC patients & $\begin{array}{c}B R C A 1 \text { exons } 2,3,5,11 \text { and } 20 \\
\text { and } B R C A 2 \text { exons } 10 \text { and } 11\end{array}$ & Dufloth, 2005 \\
\hline 402 & $9(2.3 \%)$ & $\begin{array}{l}\mathrm{BC} \text {, irrespective } \\
\text { to age or family } \\
\text { history }\end{array}$ & $\begin{array}{l}\text { Four common alterations in } \\
\text { BRCA1 and BRCA2 (185delAG, } \\
\text { 5382insC and exon } 136 \mathrm{~kb} \\
\text { duplication in BRCA1 and } \\
\text { 6174delT in BRCA2 }\end{array}$ & Gomes, 2007 \\
\hline 612 & $21(3.4 \%)$ & HBOC patients & $\begin{array}{l}\text { BRCA1 exon } 11 \text { and BRCA2 } \\
\text { exons } 10 \text { and } 11 \text { and specific } \\
\text { founder mutations }\end{array}$ & Esteves, 2009 \\
\hline 137 & $7(5 \%)$ & $\begin{array}{l}\text { non-Ashkenazyi } \\
\text { HBOC patients }\end{array}$ & $\begin{array}{l}\text { BRCA1 exon } 2 \text { (c.68_69del } \\
\text { mutation), exon } 20 \text { (c.5266dup } \\
\text { mutation) and } B R C A 2 \text { exon } 11 \\
\text { (c.5946del mutation). }\end{array}$ & Ewald, 2011 \\
\hline 255 & $3(1.2 \%)$ & $\begin{array}{l}\text { Askenazi-jews } \\
\text { unselected for } \\
\text { family history of } \\
\text { cancer }\end{array}$ & $\begin{array}{c}B R C A 1 \text { 185delAG, BRCA1 } \\
\text { 5382insC and BRCA2 6174delT }\end{array}$ & Dillenburg, 2012 \\
\hline 54 & $11(20.4 \%)$ & $\begin{array}{l}\mathrm{BC}<35 \text { years, } \\
\text { irrespective to } \\
\text { family history }\end{array}$ & $\begin{array}{l}\text { All coding regions of } B R C A 1 \\
\text { and } B R C A 2\end{array}$ & Carraro, 2013 \\
\hline 106 & $9(8.5 \%)$ & HBOC patients & $\begin{array}{l}\text { All coding regions of BRCA1 } \\
\text { and common founder mutations } \\
\text { in the BRCA } 2\end{array}$ & Felix, 2014 \\
\hline 120 & $27(22.5 \%)$ & HBOC patients & $\begin{array}{l}B R C A 1 / B R C A 2 \text { point mutations } \\
\text { and rearrangements }\end{array}$ & Silva, 2014 \\
\hline 616 & $0(0 \%)$ & $\begin{array}{l}513 \text { cancer-free } \\
\text { patients and } 103 \\
\text { OC patients }\end{array}$ & $\begin{array}{c}B R C A 1\left(\mathrm{c} .68 \_69 \mathrm{delAG},\right. \\
\text { c.5266dupC, c.181T }>\mathrm{G}, \\
\text { c.4034delA, c.5123C }>\mathrm{A}) \\
\text { and } B R C A 2(\mathrm{c} .5946 \mathrm{delT}, \\
\text { c.8537_8538delAG, } \\
493644939 \mathrm{delGAAA}, \\
\text { c.156_157insAlu) }\end{array}$ & Schayek,2015 \\
\hline 349 & $75(21.5 \%)$ & HBOC patients & $\begin{array}{c}\text { All coding regions of } B R C A 1 \\
\text { and } B R C A 2\end{array}$ & Present study \\
\hline
\end{tabular}

with this initial screening, the analysis of all coding sequence of both $B R C A 1$ and $B R C A 2$ is imperative.

The presence of c.5266dupC mutation was associated with an increased risk for OC in our series, leading to a three times higher risk than those WT individuals for that mutation. Similarly, a study conducted in Poland identified that this mutation was present in $57 \%$ of the mutated OC cases [32].

The c.5266dupC mutation represents about 98-99\% of the mutations found in Ashkenazi Jews, however it is not restricted to these populations. It has been reported as having high prevalence in several countries, mainly from Central and Eastern Europe [33]. Among the 18 patients in our study who are carriers of this mutation, although we could not assess the local ancestry of the locus containing the $B R C A 1$ c.5266dupC mutation, the European ancestry profile was prevalent in $94.4 \%$ of cases (17/18 families). For $83.3 \%$, the European contribution was higher than $69 \%$. In one case, the main contribution was the Amerindian followed by the European, demonstrating, once again, the wide miscegenation present in the Brazilian population.

Regarding genomic rearrangements, it is known that its frequency varies considerably among populations [34]. Although common in some populations, representing about $30 \%$ of the mutations identified in $B R C A 1 / B R C A 2$ genes in the Netherlands and Germany [35], large rearrangements 
are relatively rare in most populations [34]. In the present cohort, although not representative of the entire Brazilian population, only two families with rearrangements were identified, one case with rearrangement in BRCA1 (deletion of exons 5-7), and the other with a rearrangement in $B R C A 2$ gene, that is regarded as a Portuguese founder mutation (c.156_157insAlu in BRCA2), mutation that represents about $40 \%$ of deleterious germline mutations of $B R C A 1 / B R C A 2$ in Portugal $[33,36,37]$.

Concerning the variants with unknown clinical significance, we have reported a list of variants identified in our study (mostly missense variants), and, we observe a lack of consensus about their biological/clinical significance among the different existing databases. The number of cases tested in our center so far does not allow us to classify these variants based on their frequency or the co-occurrence of pathogenic mutations (although we have identified that five of our identified variants were present in individuals carrying pathogenic mutations, albeit not in the same gene for 4 of them). Therefore, our strategy consists in in silico analysis, segregation studies (which will be conducted in cases where there is availability of family members with and without cancer) and analysis of available databases. However, the analysis of databases has not been instructive in most of cases. As an example, the BRCA1 variants c.5509T $>\mathrm{C}$ and c.5096G $>$ A have been considered pathogenic by HGMD, VUS, by BIC and UMD, "probably deleterious" by LOVD, and not conclusive by ClinVar ("conflicting data"). In the case of $B R C A 2$ variants $c .4585 \mathrm{G}>\mathrm{A}$ and c. $6347 \mathrm{~A}>\mathrm{G}$, these are considered to be pathogenic by HGMD, without clinical significance by BIC and benign by ClinVar. These discrepancies between the databases, although "explicable", given the low prevalence of most of these variants, highly hinder the clinical work and the subsequent management of patients and their families. According to Miller-Samuel and colleagues "The VUS can create additional confusion and anxiety for the patient and family, and increased uncertainty for the clinician responsible for making medical management recommendations. Although clinicians are advised not to make management decisions based on VUS results, what actually happens in real-world clinical practice?" [38].

Even though clinician's decisions cannot be made based on VUS, some of our findings called our attention and deserve deep investigation. This is the case, for example for the age at diagnosis and the number of $\mathrm{BC}$ seen in the families carrying the alterations c.5425_5430delGTTGTG (BRCA1), c. $6347 \mathrm{~A}>\mathrm{G}(B R C A 2)$ and c. $1601 \mathrm{~A}>\mathrm{G}(B R C A 1)(8,5$ and $6 \mathrm{BC}$ cases respectively), as well as the very early ages at diagnosis (24 years old) of the probands with the variants c.5096A $>\mathrm{G}(B R C A 2)$ and c.6988A $>\mathrm{G}$ (BRCA2). Given the absence of consensus among databases, those families should be further evaluated through segregation analysis and functional studies. In addition, sequencing using other breast/ovarian cancer predisposition genes (high and moderate risk already present in commercial panels) should also be conducted.

It is also worth observing that the characteristic widespread miscegenation of the Brazilian population was proven in our sample. Although most of the cases analyzed present a major European ancestry component, we identified in all cases a contribution from more than one ancestor component, often equally distributed. Although study participants were from different regions of Brazil, we did not identify in this study a difference in the ancestry profile associated with the region of origin of the participants, which can be due to the high concentration of patients from the Southeast Brazilian region. According to Kedhy and colleagues [19], the African component in individuals of the northeast region of Brazil is of $50 \%$, while in the South and Southeast of Brazil, the European component exceeds $70 \%$. Still according to Kedhy and collaborators, in highly miscegenated Latin American populations such as the Brazilian, the frequency and distribution of deleterious mutations are determined more by the history of its population than for its demographics. In Brazil, for example, in 1870, Africans constituted the predominant ethnic group, which changed with the arrival of about 4 million Europeans during the second half of the 19 th century and the first half of the 20th century. These migrations transformed Brazil in a predominantly white nation, especially in the South and Southeast regions of the country [19]. Nonetheless, although we found a great predominance of European ancestry in our sample, all individuals (mutated or not) had a greater or lesser extent of ancestral profiles admixture and this mixture was not correlated with mutational status, mutation type, age at diagnosis or type of cancer developed. However, some associations or trends could be observed, such as a more aggressive cancer behavior (higher grade tumor) in patients whose African component was larger. However, these data needs to be further validated in a larger sample group.

This study has some limitations, such as the fact that other high and moderated $\mathrm{BC}$ associated genes were not included in the test. In addition, although this is the largest Brazilian study involving complete sequencing of the genes $B R C A 1 / B R C A 2$ in a high-risk sample for HBOC and correlating the findings with the ancestral profile of the population., the cohort analyzed cannot be considered representative of the whole Brazilian population and the results obtained regarding the lack of association among the mutational profile and genetic ancestry should be further validated in a bigger cohort. The genotyping of a larger sample group with all five Brazilian regions equally represented would be very informative.

In conclusion, this is the largest Brazilian study involving complete sequencing of the genes BRCA1/ $B R C A 2$ in a high-risk sample for $\mathrm{HBOC}$ and correlating the findings with the ancestral profile of the population. We identified $21.5 \%$ of $B R C A 1 / B R C A 2$ mutated patients, 
with most of the identified mutations located in the BRCA1 gene (65.3\%). Among the mutations identified, the mutations c.5266dupC and c.3331_3334delCAAG constitutes about $35 \%$ of the mutations identified in our cohort and more than $50 \%$ of the BRCA1 mutations. In addition, we could not identify any association between the ancestry profile and the distribution of BRCA1/BRCA2 mutations in the analyzed sample. Although genetic testing for these genes are already available for almost two decades, this test remains a great example of the use of human genetic variability in reducing the risk of developing cancer.

\section{MATERIALS AND METHODS}

\section{Sample population}

A total of 349 unrelated individuals at-risk for HBOC (with personal and familial history of BC and/or OC) were referred from the Oncogenetics Department (OD) for genetic testing for BRCA1/BRCA2 genes in the period between 2010 and 2014. The OD was established in 2010 at the Barretos Cancer Hospital (reference center in Brazil for the prevention and treatment of cancer) and offers multidisciplinary care and in multiple steps, ranging from genetic risk assessment to genetic testing for high-risk families identified (Palmero EI, "in press"). All families were identified by the OD and fulfilled at least one of the following criteria: a) the clinical criteria recommended by the National Institute for Health Care Excellence (NICE) [39] (for details see Supplementary Table S3); b) probability of mutation in BRCA1/BRCA2 over $20 \%$ according to the Myriad prevalence tables $[40,41]$; c) BC diagnosed below 30 years old; and d) the presence of $\mathrm{OC}$ in the family. Furthermore, in the families where a pathogenic germline mutation was identified, mutation-specific predictive genetic test was performed for all interested relatives. Confirmation of the cancer family history was attempted in all cases by pathology and medical reports and/or death certificates. The project was approved by the local Ethics in Research Committee.

\section{$B R C A 1$ and $B R C A 2$ mutation screening}

Genomic DNA was isolated from peripheral blood samples using the QIAamp DNA Blood Mini Kit (Qiagen) following the manufacturer's instructions. All coding exons were amplified by multiplex-PCR reactions, as described by Costa et al. [42].

After PCR amplification of the BRCA1 (NM_007294.3) and BRCA2 (NM_00059.3) genes, the products were purified (ExoSap-IT - USB), and sequenced (BigDye terminator v3.1 - Applied Biosystems). Following purification, samples were analyzed in a 3500xL Genetic Analyzer (Applied Biosystems). The results were analyzed using the SeqScape software (Applied Biosystems).
In addition, for samples analyzed after 2013, the sequencing of BRCA1/BRCA2 was performed using the Ion Torrent PGM platform. For this, libraries containing the PCR product of 14 multiplex PCRs were sequenced using the Ion 316 chips, which allow the simultaneous analysis of 10 to 12 patients per chip. Data analysis was performed using DNAstar Lasergene 10 software, following parameters published by Costa et al. [42] and subsequently adapted by our group to the following: (1) Q call $>=40$; (2) depth of coverage $>=100$ and, (3) SNP $\%>=23$. All the identified variants were confirmed in a new PCR reaction followed by conventional bi-directional sequencing (Sanger).

The analysis of $B R C A 1$ and $B R C A 2$ rearrangements was performed with the Multiplex Ligation-dependent Probe Amplification Kit (MLPA) according to the manufacturer's protocol. For the presence of the c.156_157ins Alu at BRCA2 gene, a mutation-specific PCR was performed following protocol described elsewhere [37].

\section{Mutations nomenclature and classification}

The nomenclature of the identified sequence variants was applied following the guidelines of the Human Genome Variation Society (HGVS) [43]. The biological significance of the variants were verified in databases such as Human Genome Mutation Database (HGMD) [44], Breast Cancer Information Core (BIC) [45], LOVD IARC [46], CLINVAR [47], Universal Mutation Database (UMD, actual BRCA Share) [48], LOVD [49] and ARUP [50]. Only mutations clearly pathogenic or previously classified as such, were included. The Align-GVGD [51], Polyphen-2 [52], SIFT [53], MAPP [54] and CADD [55] algorithms were used for the in silico analysis of the variants of unknown clinical significance identified. All variants classified as VUS or with Conflicting results by Clinvar were considered as VUSs. In addition, variants with severe disagreement (classified as pathogenic by at least one database and benign or likely benign by others) were also included. All new variants (missense, in frame and splicing), not yet reported in the other databases consulted, were also considered VUSs.

\section{Ancestry analysis}

In order to assess the genetic ancestry we employed a panel of 46 ancestry-informative markers (AIMs) selected to efficiently measure population admixture proportions of four different continental origins (African, European, East Asian and Native American) and that proved useful for the estimation of ancestral proportions in highly admixed individuals or populations like the Brazilian [56, 57]. The 46 AIMs were simply genotyped in one multiplex PCR followed by capillary electrophoresis as previously described [56]. Making use of published 
data for 748 individuals from the four continental ancestral references [56] plus 214 Brazilian control individuals previously characterized by our group [58] we then performed a comprehensive ancestry analysis in 341/349 patients. The clustering software Structure [59] was used to run supervised analyses using prior information on the origin of reference samples in order to estimate the ancestral components of the querying samples. Brazil is commonly considered as an essentially tri-hybrid (Native American, European, African) admixed population $[19,56,57,60-63]$, but there are particular locations in the country that harbor significant East Asian communities introduced more recently (e.g. São Paulo, Campinas; IBGE - Instituto Brasileiro de Geografia e Estatística, www.ibge.gov.br). For this reason, and taking into consideration the confirmed presence of individuals with East Asian ancestry among our patients, we proceeded with a conservative approach considering four possible ancestral contributors in our dataset (i.e. $\mathrm{K}=4$ ). Finally, we have performed equivalent analyses with Admixture [64] so as to assess the consistency of the ancestry estimates obtained with both programs.

\section{Statistical analysis}

Statistical analysis was conducted using SPSS v.19.0 software (Chicago, IL). The comparisons between clinical, molecular and ancestral characteristics employed a simple analyses using the Chi-square (or Fisher's exact) test. Differences in ancestry estimates between mutated and non-mutated patients were carried out using Wilcoxon rank-sum test. The association of ancestry with patient characteristics (clinical and histopathological data and family history information) were examined with Mann-Withney (or Kruskal-Wallis) tests. The level of significance considered in all tests was $5 \%$.

\section{CONFLICTS OF INTEREST}

All the authors declare no conflicts of interest.

\section{GRANT SUPPORT}

This study was partially supported by FINEP CT-INFRA (02/2010) and FAPESP (2013/24633-2). RMR is recipient of a National Council of Technological and Scientific Development (CNPq) scholarship. RP is recipient of grant SFRH/BPD/81986/2011 from the Portuguese Foundation for Science and Technology (FCT).

\section{REFERENCES}

1. INCA. (2015. Available at: www.inca.gov.br/). Portal Instituto Nacional de Câncer - INCA.
2. INCA. (2009). Manual Operacional. (Rio de Janeiro).

3. Lynch HT, Snyder C, Lynch J. Hereditary breast cancer: practical pursuit for clinical translation. Ann Surg Oncol. 2012; 196:1723-1731.

4. Acosta AX, Abe-Sandes K, Giugliani R, Bittles AH. Delivering genetic education and genetic counseling for rare diseases in rural Brazil. J Genet Couns. 2013; 226:830-834.

5. Horovitz DD, de Faria Ferraz VE, Dain S, Marquesde-Faria AP. Genetic services and testing in Brazil. J Community Genet. 2013; 43:355-375.

6. Palmero EI, Ashton-Prolla P, da Rocha JC, Vargas FR, Kalakun L, Blom MB, Azevedo SJ, Caleffi M, Giugliani R, Schuler-Faccini L. Clinical characterization and risk profile of individuals seeking genetic counseling for hereditary breast cancer in Brazil. J Genet Couns. 2007; 163:363-371.

7. Dillenburg CV, Bandeira IC, Tubino TV, Rossato LG, Dias ES, Bittelbrunn AC, Leistner-Segal S. Prevalence of 185delAG and 5382insC mutations in BRCA1, and 6174delT in BRCA2 in women of Ashkenazi Jewish origin in southern Brazil. Genet Mol Biol. 2012; 353:599-602.

8. Gomes MC, Costa MM, Borojevic R, Monteiro AN, Vieira R, Koifman S, Koifman RJ, Li S, Royer R, Zhang S, Narod SA. Prevalence of BRCA1 and BRCA2 mutations in breast cancer patients from Brazil. Breast Cancer Res Treat. 2007; 1033:349-353.

9. Ewald IP, Izetti P, Vargas FR, Moreira MA, Moreira AS, Moreira-Filho CA, Cunha DR, Hamaguchi S, Camey SA, Schmidt A, Caleffi M, Koehler-Santos P, Giugliani R, et al. Prevalence of the BRCA1 founder mutation c.5266dupin Brazilian individuals at-risk for the hereditary breast and ovarian cancer syndrome. Hered Cancer Clin Pract. 2011; 912.

10. Dufloth RM CS, Heinrich JK, et al. Analysis of BRCA1 and BRCA2 mutations in Brazilian breast cancer patients with positive family history. Sao Paulo Med J. 2005; 123192-197.

11. Esteves VF, Thuler LC, Amendola LC, Koifman RJ, Koifman S, Frankel PP, Vieira RJ. Prevalence of BRCA1 and BRCA2 gene mutations in families with medium and high risk of breast and ovarian cancer in Brazil. Braz J Med Biol Res. 2009; 425:453-457.

12. Felix GES ASC, Machado-Lopes TMB, et al. Germline mutations in BRCA1, BRCA2, CHEK2 and TP53 in patients at high-risk for HBOC: characterizing a Northeast Brazilian Population. Hum Genome Var. 2014.

13. Schayek H, De Marco L, Starinsky-Elbaz S, Rossette M, Laitman Y, Bastos-Rodrigues L, da Silva Filho AL, Friedman E. The rate of recurrent BRCA1, BRCA2, and TP53 mutations in the general population, and unselected ovarian cancer cases, in Belo Horizonte, Brazil. Cancer genetics. 2016; 2091-2 :50-52.

14. Morris GJ, Naidu S, Topham AK, Guiles F, Xu Y, McCue P, Schwartz GF, Park PK, Rosenberg AL, Brill K, Mitchell EP. Differences in breast carcinoma characteristics in newly diagnosed African-American and Caucasian patients: a single-institution compilation compared with the National 
Cancer Institute's Surveillance, Epidemiology, and End Results database. Cancer. 2007; 1104:876-884.

15. Newman LA. Breast cancer disparities: high-risk breast cancer and African ancestry. Surgical oncology clinics of North America. 2014; 233:579-592.

16. Newman LA. Disparities in breast cancer and african ancestry: a global perspective. The breast journal. 2015; 212:133-139.

17. Fejerman L, Romieu I, John EM, Lazcano-Ponce E, Huntsman S, Beckman KB, Perez-Stable EJ, Gonzalez Burchard E, Ziv E, Torres-Mejia G. European ancestry is positively associated with breast cancer risk in Mexican women. Cancer epidemiology, biomarkers \& prevention : a publication of the American Association for Cancer Research, cosponsored by the American Society of Preventive Oncology. 2010; 194:1074-1082.

18. Bonilla C, Bertoni B, Hidalgo PC, Artagaveytia N, Ackermann E, Barreto I, Cancela P, Cappetta M, Egana A, Figueiro G, Heinzen S, Hooker S, Roman E, et al. Breast cancer risk and genetic ancestry: a case-control study in Uruguay. BMC women's health. 2015; 1511.

19. Kehdy FS, Gouveia MH, Machado M, Magalhaes WC, Horimoto AR, Horta BL, Moreira RG, Leal TP, Scliar MO, Soares-Souza GB, Rodrigues-Soares F, Araujo GS, Zamudio R, et al. Origin and dynamics of admixture in Brazilians and its effect on the pattern of deleterious mutations. Proc Natl Acad Sci USA. 2015; 11228: 8696-8701.

20. Moreno-Estrada A, Gravel S, Zakharia F, McCauley JL, Byrnes JK, Gignoux CR, Ortiz-Tello PA, Martinez RJ, Hedges DJ, Morris RW, Eng C, Sandoval K, AcevedoAcevedo $\mathrm{S}$, et al. Reconstructing the population genetic history of the Caribbean. PLoS genetics. 2013; 911: e1003925.

21. Garritano S, Gemignani F, Palmero EI, Olivier M, MartelPlanche G, Le Calvez-Kelm F, Brugieres L, Vargas FR, Brentani RR, Ashton-Prolla P, Landi S, Tavtigian SV, Hainaut P, et al. Detailed haplotype analysis at the TP53 locus in p.R337H mutation carriers in the population of Southern Brazil: evidence for a founder effect. Human mutation. 2010; 312:143-150.

22. Lourenço JJ VF, Bines J, et al. BRCA1 mutations in Brazilian patients. Genet Mol Biol. 2004.

23. Silva FC, Lisboa BC, Figueiredo MC, Torrezan GT, Santos EM, Krepischi AC, Rossi BM, Achatz MI, Carraro DM. Hereditary breast and ovarian cancer: assessment of point mutations and copy number variations in Brazilian patients. BMC Med Genet. 2014; 1555.

24. Carraro DM, Koike Folgueira MA, Garcia Lisboa BC, Ribeiro Olivieri EH, Vitorino Krepischi AC, de Carvalho AF, de Carvalho Mota LD, Puga RD, do Socorro Maciel M, Michelli RA, de Lyra EC, Grosso SH, Soares FA, et al. Comprehensive analysis of BRCA1, BRCA2 and TP53 germline mutation and tumor characterization: a portrait of early-onset breast cancer in Brazil. PLoS One. 2013; 83:e57581.
25. Peixoto A, Santos C, Pinto P, Pinheiro M, Rocha P, Pinto C, Bizarro S, Veiga I, Principe AS, Maia S, Castro F, Couto R, Gouveia A, et al. The role of targeted BRCA1/BRCA2 mutation analysis in hereditary breast/ovarian cancer families of Portuguese ancestry. Clin Genet. 2015; 881:41-48.

26. Kang E, Seong MW, Park SK, Lee JW, Lee J, Kim LS, Lee JE, Kim SY, Jeong J, Han SA, Kim SW, Korean Hereditary Breast Cancer Study G. The prevalence and spectrum of BRCA1 and BRCA2 mutations in Korean population: recent update of the Korean Hereditary Breast Cancer (KOHBRA) study. Breast Cancer Res Treat. 2015; 1511:157-168.

27. Churpek JE, Walsh T, Zheng Y, Moton Z, Thornton AM, Lee MK, Casadei S, Watts A, Neistadt B, Churpek MM, Huo D, Zvosec C, Liu F, et al. Inherited predisposition to breast cancer among African American women. Breast Cancer Res Treat. 2015; 1491:31-39.

28. Weitzel JN, Clague J, Martir-Negron A, Ogaz R, Herzog J, Ricker C, Jungbluth C, Cina C, Duncan P, Unzeitig G, Saldivar JS, Beattie M, Feldman N, et al. Prevalence and type of BRCA mutations in Hispanics undergoing genetic cancer risk assessment in the southwestern United States: a report from the Clinical Cancer Genetics Community Research Network. J Clin Oncol. 2013; 312:210-216.

29. Torres D, Rashid MU, Gil F, Umana A, Ramelli G, Robledo JF, Tawil M, Torregrosa L, Briceno I, Hamann U. High proportion of BRCA1/2 founder mutations in Hispanic breast/ovarian cancer families from Colombia. Breast Cancer Res Treat. 2007; 1032:225-232.

30. Hall MJ, Reid JE, Burbidge LA, Pruss D, Deffenbaugh AM, Frye C, Wenstrup RJ, Ward BE, Scholl TA, Noll WW. BRCA1 and BRCA2 mutations in women of different ethnicities undergoing testing for hereditary breast-ovarian cancer. Cancer. 2009; 11510:2222-2233.

31. Rebbeck TR, Mitra N, Wan F, Sinilnikova OM, Healey S, McGuffog L, Mazoyer S, Chenevix-Trench G, Easton DF, Antoniou AC, Nathanson KL, Laitman Y, Kushnir A, et al. Association of type and location of BRCA1 and BRCA2 mutations with risk of breast and ovarian cancer. JAMA. 2015; 31313:1347-1361.

32. Menkiszak J, Gronwald J, Gorski B, Jakubowska A, Huzarski T, Byrski T, Foszczynska-Kloda M, Haus O, Janiszewska H, Perkowska M, Brozek I, Grzybowska E, Zientek H, et al. Hereditary ovarian cancer in Poland. Int J Cancer. 2003; 1066:942-945.

33. Janavicius R. Founder BRCA1/2 mutations in the Europe: implications for hereditary breast-ovarian cancer prevention and control. EPMA J. 2010; 13:397-412.

34. Ewald IP, Ribeiro PL, Palmero EI, Cossio SL, Giugliani R, Ashton-Prolla P. Genomic rearrangements in BRCA1 and BRCA2: A literature review. Genet Mol Biol. 2009; 323: 437-446.

35. Petrij-Bosch A, Peelen T, van Vliet M, van Eijk R, Olmer R, Drusedau M, Hogervorst FB, Hageman S, Arts PJ, Ligtenberg MJ, Meijers-Heijboer H, Klijn JG, Vasen HF, et al. 
BRCA1 genomic deletions are major founder mutations in Dutch breast cancer patients. Nat Genet. 1997; 173:341-345.

36. Machado PM, Brandao RD, Cavaco BM, Eugenio J, Bento S, Nave M, Rodrigues P, Fernandes A, Vaz F. Screening for a BRCA2 rearrangement in high-risk breast/ ovarian cancer families: evidence for a founder effect and analysis of the associated phenotypes. J Clin Oncol. 2007; 2515:2027-2034

37. Peixoto A, Santos C, Rocha P, Pinheiro M, Principe S, Pereira D, Rodrigues H, Castro F, Abreu J, Gusmao L, Amorim A, Teixeira MR. The c.156_157insAlu BRCA2 rearrangement accounts for more than one-fourth of deleterious BRCA mutations in northern/central Portugal. Breast Cancer Res Treat. 2009; 1141:31-38.

38. Miller-Samuel S, MacDonald DJ, Weitzel JN, Santiago F, Martino MA, Namey T, Augustyn A, Mueller R, Forman A, Bradbury AR, Morris GJ. Variants of uncertain significance in breast cancer-related genes: real-world implications for a clinical conundrum. Part one: clinical genetics recommendations. Semin Oncol. 2011; 384:469-480.

39. NICE. (Available at: https://www.nice.org.uk/). National Institute for Health Care Excellence.

40. Parmigiani G, Berry D, Aguilar O. Determining carrier probabilities for breast cancer-susceptibility genes BRCA1 and BRCA2. Am J Hum Genet. 1998; 621:145-158.

41. Frank TS, Deffenbaugh AM, Reid JE, Hulick M, Ward BE, Lingenfelter B, Gumpper KL, Scholl T, Tavtigian SV, Pruss DR, Critchfield GC. Clinical characteristics of individuals with germline mutations in BRCA1 and BRCA2: analysis of 10,000 individuals. J Clin Oncol. 2002; 206:1480-1490.

42. Costa JL, Sousa S, Justino A, Kay T, Fernandes S, Cirnes L, Schmitt F, Machado JC. Nonoptical massive parallel DNA sequencing of BRCA1 and BRCA2 genes in a diagnostic setting. Human mutation. 2013; 344:629-635.

43. HGVS. (Available at: www.hgvs.org/). Human Genome Variation Society. Accessed at January 2016.

44. HGMD. (Available at: http://www.biobase-international. com/product/hgmd). Human Genome Mutation Database. Accessed at January 2016.

45. BIC. (Available at: http://research.nhgri.nih.gov/bic/). Breast Cancer Information Core. Accessed at January 2016.

46. IARC L. (Available at: http://priors.hci.utah.edu/home.php). LOVD IARC. Accessed at January 2016.

47. Clinvar. Available at: www.ncbi.nlm.nih.gov/clinvar. Accessed at January 2016.

48. Share B. (Available at: http://www.umd.be/). Universal Mutation Database. Accessed at January 2016.

49. LOVD. Leiden Open Variation Database. Accessed at January 2016.

50. ARUP L.The University of Utah. Accessed at January 2016.

51. Tavtigian SV, Deffenbaugh AM, Yin L, Judkins T, Scholl T, Samollow PB, de Silva D, Zharkikh A, Thomas A.
Comprehensive statistical study of 452 BRCA1 missense substitutions with classification of eight recurrent substitutions as neutral. Journal of medical genetics. 2006; 434:295-305.

52. Polyphen. (Available at: http://genetics.bwh.harvard.edu/ pph2/). Polyphen. Accessed at January 2016.

53. SIFT. (Available at: http://sift.jcvi.org/www/SIFT_BLink_ submit.html). SIFT. Accessed at January 2016.

54. Stone EA, Sidow A. Physicochemical constraint violation by missense substitutions mediates impairment of protein function and disease severity. Genome research. 2005; 157 : 978-986.

55. Kircher M, Witten DM, Jain P, O'Roak BJ, Cooper GM, Shendure J. A general framework for estimating the relative pathogenicity of human genetic variants. Nat Genet. 2014; 463: 310-315.

56. Pereira R, Phillips C, Pinto N, Santos C, dos Santos SE, Amorim A, Carracedo A, Gusmao L. Straightforward inference of ancestry and admixture proportions through ancestry-informative insertion deletion multiplexing. PLoS One. 2012; 71:e29684.

57. Saloum de Neves Manta F, Pereira R, Vianna R, Rodolfo Beuttenmuller de Araujo A, Leite Goes Gitai D, Aparecida da Silva D, de Vargas Wolfgramm E, da Mota Pontes I, Ivan Aguiar J, Ozorio Moraes M, Fagundes de Carvalho E, Gusmao L. Revisiting the genetic ancestry of Brazilians using autosomal AIM-Indels. PLoS One. 2013; 89:e75145.

58. Campanella NC, Berardinelli GN, Scapulatempo-Neto C, Viana D, Palmero EI, Pereira R, Reis RM. Optimization of a pentaplex panel for MSI analysis without control DNA in a Brazilian population: correlation with ancestry markers. Eur J Hum Genet. 2014; 227:875-880.

59. Pritchard JK, Stephens M, Donnelly P. Inference of population structure using multilocus genotype data. Genetics. 2000; 1552:945-959.

60. Pena SD, Di Pietro G, Fuchshuber-Moraes M, Genro JP, Hutz MH, Kehdy Fde S, Kohlrausch F, Magno LA, Montenegro RC, Moraes MO, de Moraes ME, de Moraes MR, Ojopi EB, et al. The genomic ancestry of individuals from different geographical regions of Brazil is more uniform than expected. PLoS One. 2011; 62:e17063.

61. Moura RR, Coelho AV, Balbino Vde Q, Crovella S, Brandao LA. Meta-analysis of Brazilian genetic admixture and comparison with other Latin America countries. American journal of human biology. 2015; 275:674-680.

62. Lima-Costa MF, Rodrigues LC, Barreto ML, Gouveia M, Horta BL, Mambrini J, Kehdy FS, Pereira A, RodriguesSoares F, Victora CG, Tarazona-Santos E, Epigen-Brazil g. Genomic ancestry and ethnoracial self-classification based on 5,871 community-dwelling Brazilians (The Epigen Initiative). Scientific reports. 2015; 59812.

63. Santos HC, Horimoto AV, Tarazona-Santos E, RodriguesSoares F, Barreto ML, Horta BL, Lima-Costa MF, 
Gouveia MH, Machado M, Silva TM, Sanches JM, Esteban N, Magalhaes WC, et al. A minimum set of ancestry informative markers for determining admixture proportions in a mixed American population: the Brazilian set. Eur J Hum Genet. 2015.

64. Alexander DH, Novembre J, Lange K. Fast model-based estimation of ancestry in unrelated individuals. Genome research. 2009; 199:1655-1664.
65. Dufloth RM, Carvalho S, Heinrich JK, Shinzato JY, dos Santos CC, Zeferino LC, Schmitt F. Analysis of BRCA1 and BRCA2 mutations in Brazilian breast cancer patients with positive family history. Sao Paulo Med J. 2005; 1234 : 192-197. 